\title{
CHARACTERIZING THE POPULATION OF BRIGHT INFRARED SOURCES IN THE SMALL MAGELLANIC CLOUD
}

\author{
K. E. Kraemer ${ }^{1}$, G. C. Sloan ${ }^{2,3,4}$, P. R. Wood ${ }^{5}$, O. C. Jones ${ }^{4}$, and M. P. Egan ${ }^{6}$ \\ ${ }^{1}$ Institute for Scientific Research, Boston College, 140 Commonwealth Avenue, Chestnut Hill, MA 02467, USA; kathleen.kraemer@bc.edu \\ ${ }^{2}$ Center for Astrophysics and Planetary Science, Cornell University, Ithaca, NY 14853-6801, USA; sloan@astro.cornell.edu \\ ${ }^{3}$ Department of Physics and Astronomy, University of North Carolina, Chapel Hill, NC 27599-3255, USA \\ ${ }^{4}$ Space Telescope Science Institute, 3700 San Martin Drive, Baltimore, MD 21218, USA \\ ${ }^{5}$ Research School of Astronomy and Astrophysics, Australian National University, Cotter Road, Weston Creek ACT 2611, Australia; wood@mso.anu.edu.au \\ ${ }^{6}$ National Geospatial Intelligence Agency, 7500 GEOINT Drive, Springfield, VA 22150, USA; michael.p.egan@ nga.mil \\ Received 2016 September 15; revised 2016 October 13; accepted 2016 October 14; published 2017 January 12
}

\begin{abstract}
We have used the Infrared Spectrograph (IRS) on the Spitzer Space Telescope to observe stars in the Small Magellanic Cloud (SMC) selected from the Point Source Catalog of the Midcourse Space Experiment (MSX). We concentrate on the dust properties of the oxygen-rich evolved stars. The dust composition has smaller contributions from alumina compared to the Galaxy. This difference may arise from the lower metallicity in the SMC, but it could be a selection effect, as the SMC sample includes more stars that are brighter and thus more massive. The distribution of the SMC stars along the silicate sequence looks more like the Galactic sample of red supergiants than asymptotic giant branch stars (AGBs). While many of the SMC stars are definitively on the AGB, several also show evidence of hot bottom burning. Three of the supergiants show PAH emission at $11.3 \mu \mathrm{m}$. Two other sources show mixed chemistry, with both carbon-rich and oxygen-rich spectral features. One, MSX SMC 134, may be the first confirmed silicate/carbon star in the SMC. The other, MSX SMC 049, is a candidate post-AGB star. MSX SMC 145, previously considered a candidate OH/IR star, is actually an AGB star with a background galaxy at $z=0.16$ along the same line of sight. We consider the overall characteristics of all the MSX sources, the most infrared-bright objects in the SMC, in light of the higher sensitivity and resolution of Spitzer, and compare them with the object types expected from the original selection criteria. This population represents what will be seen in more distant galaxies by the upcoming James Webb Space Telescope (JWST). Color-color diagrams generated from the IRS spectra and the mid-infrared filters on JWST show how one can separate evolved stars from young stellar objects (YSOs) and distinguish among different classes of YSOs.
\end{abstract}

Key words: circumstellar matter - infrared: stars - Magellanic Clouds

Supporting material: machine-readable tables

\section{INTRODUCTION}

The proximity of the Small Magellanic Cloud (SMC) makes it possible to study in detail the various components of a galactic ecosystem that fundamentally differs from our own Galaxy. The SMC has a comparatively low metallicity, [Fe/H] $\sim-0.6$ to -0.8 (Keller \& Wood 2006, and references therein) and a mean distance modulus of $18.91 \pm 0.02$ (Rubele et al. 2015). This gives us a nearby analog of the metal-poor systems that populate the early universe.

Naked stars or stars with optically thin circumstellar material dominate the samples observed in the optical and near-infrared, but in the past $\sim 15-20$ years, mid-infrared maps have revealed the population of cooler and more embedded objects. To identify and characterize the various populations, we embarked on a program to obtain infrared spectra of representative sources with the Spitzer Space Telescope (Werner et al. 2004) using the Infrared Spectograph (IRS; Houck et al. 2004). These spectra can test the population characteristics inferred from the photometric data.

When the Spitzer mission began in 2003, the best available mid-infrared point source catalog of the SMC was from the Midcourse Space Experiment (MSX), launched by the U.S. Air Force in 1996. On board, the Spatial Infrared Imaging Telescope (SPIRIT III) had four filters centered at 8.3, 12.1, 14.7, and $21.3 \mu \mathrm{m}$ (named A, C, D, and E, respectively). The astronomical observations during the cryogenic part of the mission, described by Price et al. (2001), included surveys of the Galactic plane (Price et al. 2001), the Large Magellanic Cloud (LMC; Egan et al. 2001), and the SMC, as well as Galactic star-forming regions (e.g., Kraemer et al. 2003), other external galaxies (e.g., Kraemer et al. 2002a), and the gaps in coverage of the Infrared Astronomical Satellite (IRAS; Neugebauer et al. 1984; Price et al. 2001).

The SMC catalog from MSX consists of the 243 sources in the main MSX catalog (Egan et al. 2003) that lie within the region $7.0<\alpha<18.7$ and $-74.4<\delta<-71.5$. This catalog reveals the bright mid-infrared population of the SMC. All 243 sources were detected in Band A, the most sensitive band, with a small number of sources also detected in the other bands. An additional 63 sources are in the lower signal-to-noise ratio $(\mathrm{S} / \mathrm{N})$ catalog $(\mathrm{S} / \mathrm{N} \sim 3-5)$ with Band $\mathrm{A}$ detections and can readily be seen in the image, even though they did not meet the $\mathrm{S} / \mathrm{N}$ criteria for the main catalog. The observed Band A magnitudes ${ }^{7}$ ranged from $\sim 3.0$ to 8.2 ( $\sim 0.03-3.75 \mathrm{Jy})$, down to $8.8 \mathrm{mag}(0.017 \mathrm{Jy})$ for the low $\mathrm{S} / \mathrm{N}$ set. In comparison, the other mid-IR catalog available when Spitzer launched, the IRAS Point Source Catalog (Beichman et al. 1988), contains $\sim 50$ point sources of $\sim 0.2-3 \mathrm{Jy}$ in the SMC (including the Faint Source Catalog).

\footnotetext{
The zero magnitude flux for Band A of MSX is $58.5 \mathrm{Jy}$.
} 
Table 1

Observing Log

\begin{tabular}{|c|c|c|c|c|c|c|c|}
\hline \multirow{2}{*}{$\begin{array}{l}\text { MSX } \\
\text { SMC }\end{array}$} & \multirow{2}{*}{ R.A. } & \multirow[b]{2}{*}{ (J2000.0) } & \multirow[b]{2}{*}{ PID } & \multirow[b]{2}{*}{ AOR } & \multicolumn{2}{|c|}{$t_{\text {int }}(\mathrm{s})$} & \multirow{2}{*}{$\begin{array}{c}\text { Obs. date } \\
\left(\mathrm{MJD}^{\mathrm{a}}\right)\end{array}$} \\
\hline & & & & & $\overline{\mathrm{SL}}$ & $\overline{\mathrm{LL}}$ & \\
\hline 000 & 005518.00 & -720532.0 & 3277 & 10666240 & 240 & 1440 & 53303.5 \\
\hline 018 & 004631.59 & -732846.4 & 3277 & 10668800 & 112 & 168 & 53301.2 \\
\hline 024 & 004252.23 & -735051.7 & 3277 & 10663169 & 168 & 240 & 53521.8 \\
\hline 055 & 005007.19 & -733125.2 & 3277 & 10657536 & 112 & 240 & 53303.7 \\
\hline 067 & 004736.89 & -730444.2 & 3277 & 10664192 & 240 & 1440 & 53304.2 \\
\hline 096 & 005006.40 & -732811.2 & 3277 & 10667008 & 168 & 1200 & 53482.7 \\
\hline 109 & 005129.68 & $\begin{array}{lll}-73 & 10 & 44.2\end{array}$ & 3277 & 10667520 & 112 & 1440 & 53484.7 \\
\hline 134 & 005044.40 & -723739.0 & 3277 & 10665216 & 240 & 960 & 53304.1 \\
\hline 149 & 010938.24 & $\begin{array}{lll}-73 & 20 & 02.4\end{array}$ & 3277 & 10668032 & 112 & 960 & 53303.0 \\
\hline 168 & 005526.76 & -723556.1 & 3277 & 10668288 & 240 & 1440 & 53304.1 \\
\hline 181 & 010048.17 & -725102.1 & 3277 & 10665728 & 112 & 960 & 53303.0 \\
\hline 049 & 004452.56 & $\begin{array}{lll}-73 & 18 & 25.9\end{array}$ & 30355 & 17408768 & 60 & 240 & 53914.6 \\
\hline 101 & 004851.82 & $\begin{array}{lll}-73 & 22 & 39.9\end{array}$ & 30355 & 17409024 & 60 & 360 & 53914.6 \\
\hline 145 & $0052 \quad 12.93$ & -730853.0 & 30355 & 17409280 & 60 & 240 & 53914.6 \\
\hline 161 & 010810.32 & -731552.4 & 30355 & 17409536 & 60 & 240 & 53914.7 \\
\hline
\end{tabular}

Note.

${ }^{\mathrm{a}}$ (JD-2400000.5).

(This table is available in machine-readable form.)

This paper focuses on the IRS observations of the SMC sources in the MSX catalog, with an emphasis on the objects with oxygen-rich spectra in the infrared. In Section 2, we describe how we selected the sources and produced the IRS spectra presented here. Section 3 describes the oxygen-rich evolved stars and their dust properties. We assess the photometric diagrams and discuss the results in Section 4. Individual sources with peculiar properties are described in Section 5. The Appendix describes how we have revised the MSX SMC catalog, using the recent Spitzer surveys of the SMC to improve the positional accuracy for the source positions and expand our photometric knowledge of these sources.

\section{OBSERVATIONS AND DATA REDUCTION}

\subsection{Creating the Sample}

The 243 MSX SMC sources were matched with nearinfrared data from the Two Micron All-Sky Survey (2MASS; Cutri et al. 2003; Skrutskie et al. 2006) assuming that the 2MASS source closest to the MSX coordinates was in fact the appropriate association. We then chose a sample of targets to span the range of colors in $J-K_{s}$ and $K_{s}-A$ that cover the expected positions of evolved stars, based on previous efforts that used model spectra to classify sources in the MSX catalog of the LMC (Egan et al. 2001).

Our original set of infrared spectra of MSX SMC sources was obtained in Spitzer Cycle 1 (Program ID 3277, P.I. M. Egan). This program included 35 targets from the MSX SMC catalog and one additional target known to be oxygen-rich from its optical spectrum ${ }^{8}$ (Smith et al. 1995). These targets include 19 carbon-rich stars on the asymptotic giant branch (AGB; Sloan et al. 2006b, 2016), two R CrB candidates (Kraemer et al. 2005), one carbon-rich post-AGB object (Kraemer et al. 2006), and two young stellar objects (YSOs; Oliveira

\footnotetext{
8 HV 12122 can be seen on the MSX Band A image but was below the S/N threshold for inclusion in the catalog. This source was observed as MSX SMC 000
}

et al. 2013). This paper examines the remaining 11 sources in the sample. ${ }^{9}$ Nine of these are evolved stars with oxygen-rich properties in the mid-infrared (see also Jones et al. 2012, 2014), and the last two are largely featureless. Additionally, two of the spectra from PID 3277 showed particularly strong crystalline silicate features. We subsequently selected four objects in the MSX SMC catalog with similar photometric characteristics in an effort to uncover additional sources with crystalline dust. We observed these targets in Spitzer Cycle 3 (Program ID 30355 , P.I. J. Houck), and three of the spectra show unusual spectral features, including two with both oxygen- and carbonrich features.

Table 1 provides particulars about the observations, including the coordinates from the photometric matching described in the Appendix. Table 2 presents the basic properties of the 11 oxygen-rich objects from Cycle 1 and the 4 from Cycle 3, including bolometric magnitudes, infrared spectral classifications, and object classes, which are explained below.

Table 3 lists 20 additional MSX SMC sources that were observed by other IRS programs. Overall, 59 MSX SMC sources were observed with the IRS. Although Sections 2 and 3 concentrate on the sources in Table 1, all 59 are considered in the photometric analysis in Section 4. We refer the reader to the papers in the table references for further details on the additional objects.

\subsection{Observations}

The spectra were observed using the low-resolution modules of the IRS, Short-Low (SL) and Long-Low (LL), which provided spectra in the 5-14 and 14-37 $\mu \mathrm{m}$ ranges, respectively, at a resolution between $\sim 60$ and 120 . The spectrum in each module includes a second-order and first-order segment obtained in separate apertures. The observations were made with the standard staring mode, which placed each target at positions one-third and two-thirds along the slit. Thus, a full low-resolution spectrum combines data from eight separate

\footnotetext{
No data were obtained for one target due to a typo in the input coordinates.
} 
Table 2

Observed Program Sources

\begin{tabular}{|c|c|c|c|c|c|c|c|c|c|}
\hline \multirow{2}{*}{$\begin{array}{l}\text { MSX } \\
\text { SMC }\end{array}$} & \multirow[b]{2}{*}{ Source name } & \multicolumn{2}{|c|}{ Period } & \multicolumn{2}{|c|}{ Optical } & \multirow{2}{*}{$\begin{array}{l}\text { Infrared } \\
\text { Sp. Class }\end{array}$} & \multirow[b]{2}{*}{$M_{\mathrm{bol}}$} & \multirow{2}{*}{$\begin{array}{l}\text { R15 } \\
\text { Class }^{c}\end{array}$} & \multirow{2}{*}{$\begin{array}{l}\text { Var. } \\
\text { Type }^{\mathrm{d}}\end{array}$} \\
\hline & & (days) & References $^{\mathrm{a}}$ & Sp. Type & References $^{\mathrm{b}}$ & & & & \\
\hline 000 & HV 12122 & 545 & G09 & M4-5.5 II/III & P83, G15 & 1.N: & -7.01 & O-EAGB & M: \\
\hline 018 & 2M J00463159 & 915 & G09 & M & K16 & 2.SE6xf & -6.39 & O-AGB & $\cdots$ \\
\hline 024 & HV 1375 & 400 & S11 & M5 II & W83, G15 & 2.SE5xf: & -6.27 & O-AGB & M \\
\hline 055 & IRAS $00483-7347$ & 1859 & S11 & M & G98 & 2.SE7f & -7.54 & $\mathrm{O}-\mathrm{AGB}^{\mathrm{c}}$ & LC: \\
\hline 067 & HV 11262 & $\cdots$ & & K2.5-M3 I-Iabe & Multiple & $1 . \mathrm{N}$ & -7.86 & RSG & LC: \\
\hline 096 & PMMR 34 & $\cdots$ & & K3-M1 I-Iab & Multiple & 2.SE3u & -7.70 & RSG & $\cdots$ \\
\hline 109 & PMMR 41 & $\cdots$ & & K3-M1.5 I-Ib & Multiple & 2.SE7u & -8.03 & RSG & $\mathrm{LC}$ \\
\hline 134 & RAW 631 & 141 & S11 & $\mathrm{C}$ & R93, R05, K16 & $2 . \mathrm{SX} 6$ & -5.04 & $\mathrm{O} / \mathrm{C}-\mathrm{AGB}^{\mathrm{c}}$ & $\cdots$ \\
\hline 149 & HV 2084 & 545 & P97 & K5-M4 I-Iab & Multiple & 2.SE7 & -8.44 & RSG & LC: \\
\hline 168 & HV 1652 & $\cdots$ & & K2-M0/1 I-Iab & Multiple & 2.SE8 & -7.79 & RSG & $\mathrm{LC}$ \\
\hline 181 & HV 11417 & 1092 & S11 & M4-5 Ie & $\mathrm{E} 80, \mathrm{P} 83$ & 2.SE7 & -7.02 & $\mathrm{O}-\mathrm{AGB}^{\mathrm{c}}$ & $\mathrm{SRc}$ \\
\hline 049 & 2M J00445256 & 158 & $\mathrm{~S} 11$ & $\mathrm{C}$ & K16 & 3.C/SX & -5.13 & pAGB: $:^{c}$ & $\cdots$ \\
\hline 101 & PMMR 24 & 213 & P97 & K2-M2 I-Iab & Multiple & 2.SE4u & -7.61 & RSG & $\cdots$ \\
\hline 145 & BMB-B 75 & 761 & S11 & M6 & B80 & 3.SE8+5.Uez & -6.28 & $\mathrm{O}-\mathrm{AGB}+\mathrm{hi}-\mathrm{z}^{\mathrm{c}}$ & SR \\
\hline 161 & IRAS F01066-7332 & 882 & $\mathrm{~S} 11$ & M & G98 & 3.SE7 & -6.02 & O-AGB & $\ldots$ \\
\hline
\end{tabular}

Notes.

a Period references: P97, Pojmański (1997, 2014), G09, Groenewegen et al. (2009), S11, Soszyński et al. (2011)

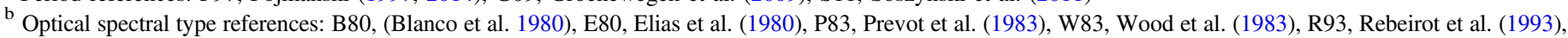

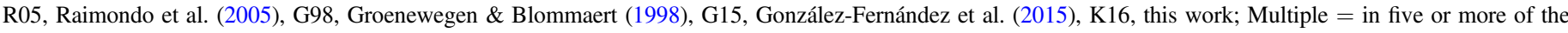
references. Additional, multiply used references: Sanduleak (1989), Elias et al. (1985), Massey \& Olsen (2003), Levesque et al. (2006).

${ }^{c}$ R15 Class: class from Ruffle et al. (2015), with the marked sources changed as discussed in the text.

d Variability class from the GCVS (Samus et al. 2009)

(This table is available in machine-readable form.)

pointings of the telescope (two modules, two apertures, two nod positions).

For 10 evolved stars with oxygen-rich dust in our Cycle 1 program, we obtained spectra from 0.45 to $1.03 \mu \mathrm{m}$ with the Double-Beam Spectrograph at the $2.3 \mathrm{~m}$ telescope of the Australian National University at Siding Spring Observatory. A $0.45-0.89 \mu \mathrm{m}$ spectrum for one of the stars in program 30355 was also observed. These spectra have a resolution of $10 \AA$, and they were reduced with standard IRAF ${ }^{10}$ procedures using the giant star HD 26169 to remove telluric features and HR 718 as a photometric standard.

\subsection{Data Reduction}

To reduce the IRS spectra, we used the Cornell spectral pipeline, which starts with the basic calibrated data, twodimensional flatfielded images produced by the S18.18 reduction pipeline from the Spitzer Science Center (SSC). The next step differences images to remove background emission and other additive offsets. For the SL data, the data with the source in the same nod position, but in the other aperture, served as the background image. (This method is known as aperture differencing.) For LL, we used data with the source in the opposite nod in the same aperture as the background image (nod differencing). We adhered to these rules unless background gradients or additional sources in the slits forced us to search for a more suitable background image. Differenced images were then cleaned using the rogue pixel masks supplied by the SSC and the imclean pixel-replacement algorithm developed at Cornell. ${ }^{11}$ Spectra were extracted from the co-added images using the optimal extraction method described by Lebouteiller et al. (2010).

\footnotetext{
${ }_{10}^{10}$ Image Reduction and Analysis Facility

11 Available as part of the irsclean package from the SSC.
}

We used spectral corrections generated from observations of HR 6348 (K0 III) for SL, and HR 6348 and HD 173511 (K5 III) for LL, to calibrate the data photometrically, as described by Sloan et al. (2015). We applied this calibration separately to each of the eight individual spectral pieces. When combining the spectra from two nods in the same order, we used a sigmaclipping algorithm to remove spikes or divots appearing only in one nod. At this point, we recalculated the spectrophotometric uncertainties by comparing the spectra from the two nods.

The above steps result in four calibrated spectra, from both spectral orders in both modules, for each star. To combine these, we multiplicatively scale the segments upwards to what is presumably the best-centered segment and trim the overlapping data. These corrections are usually less than $10 \%$, but they can be larger, especially if a source is spatially extended compared to the 3." 6 SL slit. The corrections to MSX SMC 049 and 161 were slightly over $15 \%$ in one of the two SL modules, but not the other. One of the LL nods for MSX SMC 067 was contaminated by a red source in the slit, so only data from the other nod were used.

\subsection{Basic Stellar Properties}

Table 2 includes the pulsation period and the optical spectral type if known, the infrared spectral classification and object type, the bolometric magnitude, and the variable star type from the General Catalog of Variable Stars (GCVS; Samus et al. 2009).

The pulsation periods are largely from studies using data from the Optical Gravitational Lensing Experiment (OGLE; Soszyński et al. 2011), and show a surprising amount of variation from study to study. Some of the disagreement can be attributed to the variability types (the last column in Table 2), as only two sources are Mira variables. Multiple pulsation 
Table 3

MSX SMC Targets Observed in Other Programs

\begin{tabular}{|c|c|c|c|c|c|c|c|}
\hline $\begin{array}{l}\text { MSX } \\
\text { SMC }\end{array}$ & Source name & $\begin{array}{l}\text { Prog. } \\
\text { ID }\end{array}$ & $\begin{array}{l}\text { AOR } \\
\text { key }\end{array}$ & $\begin{array}{l}\text { Infrared } \\
\text { Sp. Class }\end{array}$ & $\begin{array}{l}\text { R15 } \\
\text { Class }\end{array}$ & $M_{\mathrm{bol}}$ & References \\
\hline 027 & GM 780 & 3505 & 12931072 & 2.CE2 & C-AGB & -5.86 & L07 \\
\hline 039 & PMMR 23 & 50167 & 25689856 & $1 . \mathrm{N}$ & RSG & -7.73 & $\mathrm{~J} 12$ \\
\hline 041 & SMP SMC 006 & 103 & 4954112 & 4.Ue & C-PN & -4.82 & BS08, BS09, S14 \\
\hline 057 & LIN 60 & 50240 & 27524608 & 5.SAuei & YSO-1 & -7.24 & $\mathrm{O} 13$ \\
\hline 072 & PMMR 19 & 50167 & 25689600 & 2.SE6 & RSG & -8.41 & $\mathrm{~J} 12$ \\
\hline 099 & S3MC 170098 & 50240 & 27518720 & 4.SAi & YSO-1 & -5.71 & $\mathrm{O} 13$ \\
\hline 100 & SMP SMC 011 & 103 & 15902976 & 5.Ue & C-PN & -5.80 & S14 \\
\hline 102 & IRAS $00554-7351$ & 3505 & 12936192 & 3.CE3 & C-AGB & -6.00 & L07 \\
\hline 104 & S3MC 170445 & 50240 & 27518208 & 4.SAi & YSO-1 & -6.06 & $\mathrm{O} 13$ \\
\hline 107 & [MA 93] 226 & 50240 & 27524864 & 5.Eui & YSO-1 & -5.72 & $\mathrm{O} 13$ \\
\hline 128 & ISO $00549-7303$ & 3505 & 12932608 & 3.CE3 & C-AGB & -5.20 & L07 \\
\hline 133 & LHA $115-\mathrm{N} 32$ & 50240 & 27534080 & 5.Ue & YSO-3 & -5.57 & LK16 \\
\hline 138 & PMMR 52 & 3505 & 12931584 & 2.SE5 & RSG & -8.05 & L07 \\
\hline 153 & LIN 250 & 50240 & 27523584 & 3.SE4x & $\mathrm{B}[\mathrm{e}]$ star & -6.20 & $\mathrm{R} 15$ \\
\hline 185 & LIN 254 & 50240 & 27523072 & 4.SE3 & symbiotic & -5.31 & O13, R15 \\
\hline 191 & LHA 115-N 61 & 50240 & 27530240 & 5.Ue & H II & -5.94 & LK16 \\
\hline 199 & IRAS $00562-7255$ & 50240 & 27541248 & 5.Uei & YSO-1 & -4.77 & $\mathrm{O} 13$ \\
\hline 203 & NGC 419 MIR 1 & 3505 & 12934400 & 3.CE5 & C-AGB & -4.92 & L07 \\
\hline 210 & HV 12149 & 200 & 6019328 & 2.SE8 & O-AGB & -7.03 & S08 \\
\hline 234 & LHA 115-S 38 & 50240 & 27522048 & 3.SE7u: & O-PAGB & -5.52 & $\mathrm{R} 15$ \\
\hline
\end{tabular}

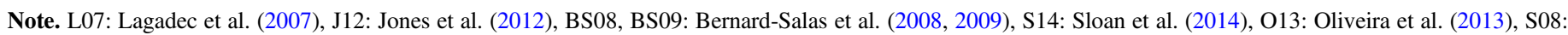
Sloan et al. (2008), LK16: L. D. Keller et al. (2016, in preparation), R15: Ruffle et al. (2015)

(This table is available in machine-readable form.)

modes are typically present in semi-regular variables, so more than one period can often be fit to a light curve (OGLE reports up to three). To decide among the different periods (Groenewegen 2004; Groenewegen et al. 2009; Soszyński et al. 2011), we examined the light curves with Vizier's online Javascript tool and selected the period that gave what appeared to be the best folded light curve. Typically (in six cases), this was the primary period given by Soszyński et al. (2011), but in three cases we selected that of Groenewegen et al. (2009). For two objects without OGLE data, we used the periods determined by the All Sky Automated Survey (Pojmański 1997, 2014).

The optical spectral types also vary depending on the investigator. Some of that is probably due to different spectral resolution or details in the line analysis. Some of the differences are real, reflecting the changes a star undergoes during its pulsation cycle. For example, González-Fernández et al. (2015) found a slightly different spectral type for each of up to three observational epochs in their sample of RSGs. Here, we try to include the range of spectral types that have been found, and the table references indicate which studies contribute to the types for each star.

The bolometric magnitudes were calculated by integrating through the photometry in the revised MSX SMC catalog (see the Appendix) and the IRS data. In some cases, the photometry extends to wavelengths as short as the $B$ band. A $3600 \mathrm{~K}$ blackbody is used to extrapolate to the blue of the blue-most photometric point, and a Rayleigh-Jeans tail is used to extrapolate from the red end of the IRS data. We assume an average distance modulus of DM $=18.9$ (Rubele et al. 2015).

The infrared spectral classifications follow the Hanscom method, as described by Kraemer et al. (2002b) and revised by Sloan et al. $(2008,2016)$ for spectra from the IRS. In this system, the numbered group indicates the overall color of the spectral energy distribution in the mid-infrared, from 1 for blue sources with stellar continua to 5 to very red sources with cool dust emission. The letters reflect the dominant spectral features, usually SE for silicate emission in this sample. Other types used in Table 2 include: $\mathrm{N}$ (naked or dust free), $\mathrm{C}$ (carbonrich), SX (crystalline silicates), and U (unidentified infrared). One source, MSX SMC 145, shows a composite spectrum, and we have classified the components separately (Section 5.4).

The object types are generally based on ancillary data, not just the IRS spectrum. They are primarily from Ruffle et al. (2015), with modifications for a few objects described in Sections 3.2 and 5.

\section{RESULTS}

\subsection{Dust Properties}

Figure 1 shows the (normalized) IRS spectra of the 15 sources from programs 3277 and 30355 with infrared or optical spectral features consistent with an oxygen-rich chemistry, either in the dust or the photosphere. The estimated $8 \mu \mathrm{m}$ flux for Band 4 of the Infrared Array Camera (IRAC; Fazio et al. 2004) is given next to the name of each source. Figures 2 and 3 present the optical spectra for the sources from program 3277, as well as for MSX SMC 049. Those with oxygen-rich photospheric features are in Figure 2, while the two sources with carbon-rich features in the optical are in Figure 3. Because MSX SMC 049 has both oxygen-rich and carbon-rich features in its IRS spectrum (Section 5.3), it is excluded from the analysis of the oxygen-rich dust. While MSX SMC 134 has the optical spectrum of a carbon star, its dust chemistry in the infrared is oxygen-rich, so it is included here.

To characterize the properties of the oxygen-rich dust, we extract from the spectra several parameters that were originally defined by Sloan \& Price $(1995,1998)$ and updated by Sloan et al. (2008). We assumed a $3600 \mathrm{~K}$ Planck function for the star, fitted to the spectrum in the 6.8-7.4 $\mu \mathrm{m}$ interval. This 


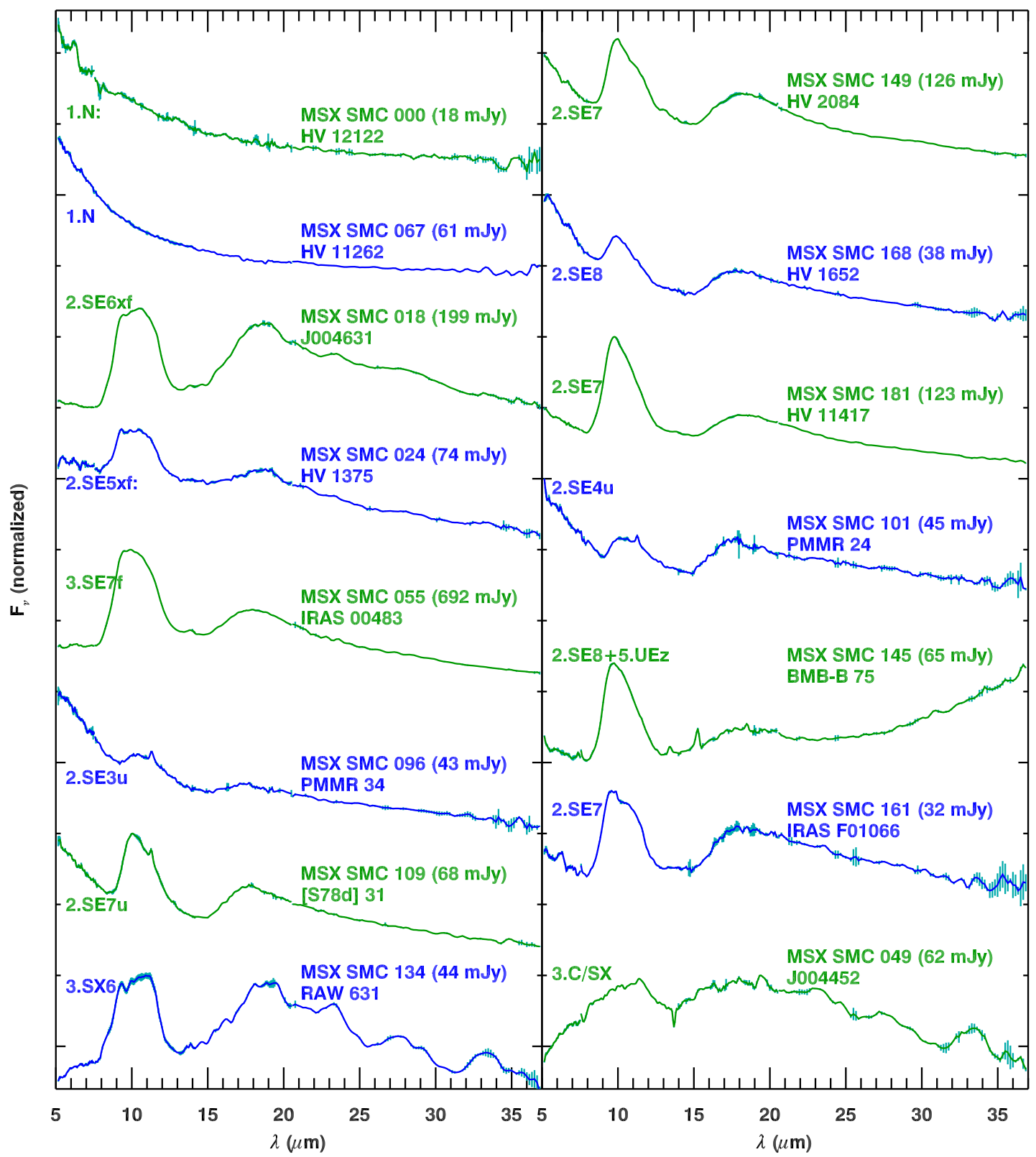

Figure 1. IRS spectra of the 15 oxygen-rich evolved stars in programs 3277 and 30355 . The flux in parenthesis is the equivalent flux for the IRAC $8 \mu \mathrm{m}$ band.

continuum differs from that assumed for Galactic stars by Sloan \& Price (1995), who used a 3240 K Engelke function (Engelke 1992) with $15 \% \mathrm{SiO}$ absorption at $8 \mu \mathrm{m}$. In the LMC and SMC, however, Sloan et al. (2008) found that the SiO absorption was much weaker in the evolved stars. For sources with strong dust features, the choice between these two stellar continua has little impact on the dust-related parameters in Table 4.

The [7]-[15] color gives a synthetic color from two narrow bands between the dust and molecular features (Sloan et al. 2008). Models by Groenewegen et al. (2009) have shown that the [7]-[15] color correlates with mass-loss rate, assuming similar outflow velocities and gas-to-dust ratios. The remaining columns in the table quantify the strength and shape of the dust emission features and follow the definitions of Sloan \& Price (1995). We assume that the dust is optically thin and subtract the fitted stellar continuum to isolate the residual dust emission. The dust emission contrast (DEC) is the ratio of stellar continuum to dust excess from 7.67 to $14.03 \mu \mathrm{m}$. The strength of the dust excess in narrow windows at 10,11 , and $12 \mu \mathrm{m}$ gives the flux ratios $F_{10} / F_{12}$ and $F_{10} / F_{11}$. Plotting these ratios produces the "silicate dust sequence," which can be modeled with a simple power law (Figure 4). Shifting the flux ratios for a given spectrum to the closest point on the silicate dust sequence gives the corrected flux ratio $F_{11} / F_{12}$ in Table 4 .

This ratio, $F_{11} / F_{12}$, in turn, leads to the silicate emission index, ${ }^{12}$ which we use to classify the dust composition (last column of the table). Egan \& Sloan (2001) found that spectra dominated by amorphous alumina-rich dust have classifications of SE1-3, while silicate-dominated spectra are SE6-8. The SE3-6 range is more difficult to characterize; it could arise from optically thick silicate emission starting to show selfabsorption, and/or more crystalline dust (e.g., Sloan et al. 2006a; Guha Niyogi et al. 2011). The SE5 and 6 sources

$\overline{{ }^{12} \mathrm{SE} \text { index } \doteq \text { floor(corrected } 10^{*} F_{11} / F_{12}-7.5}$. 

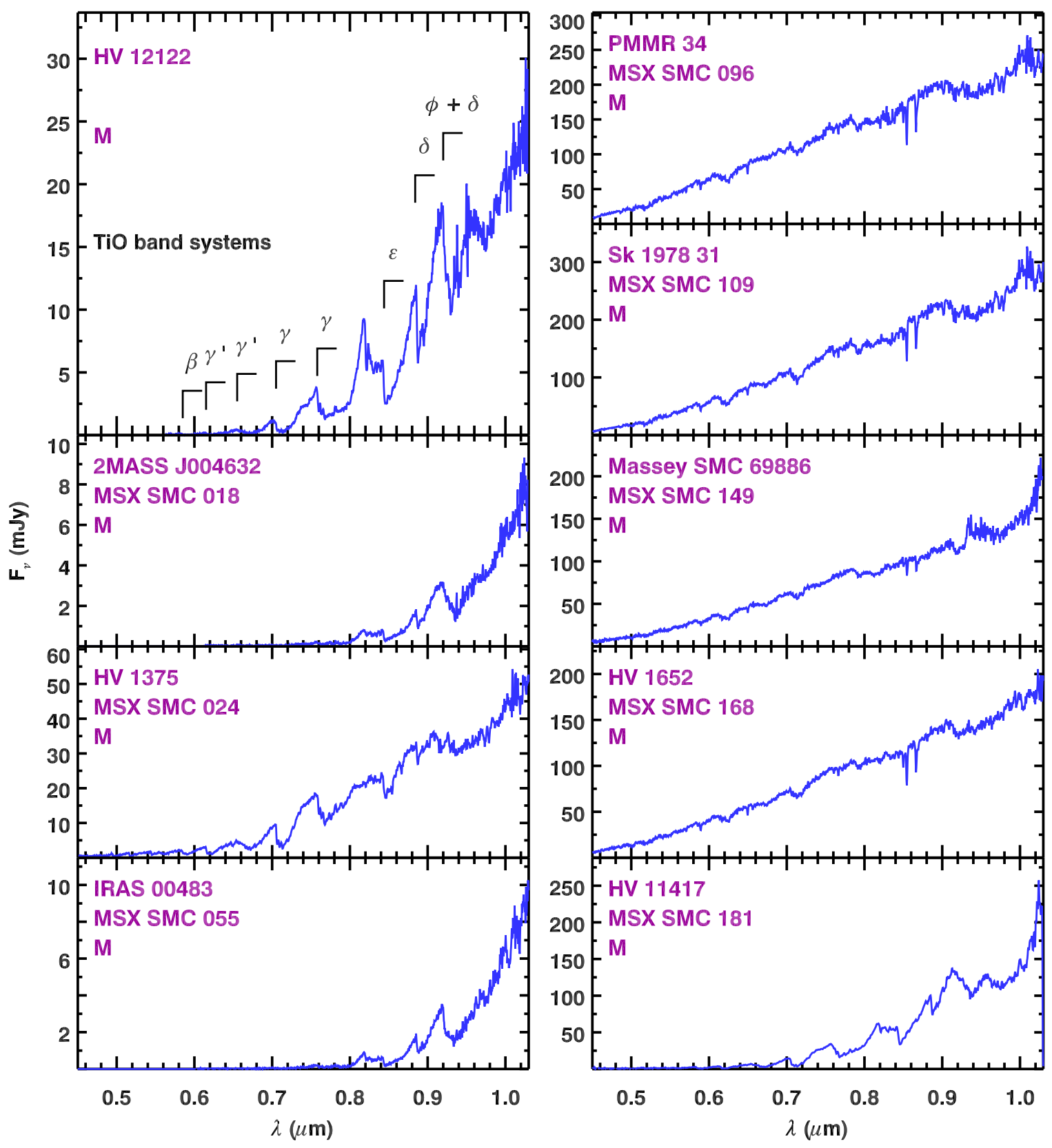

Figure 2. Optical spectra of the oxygen-rich evolved stars in program 3277.

in this sample both have weak crystalline features (MSX SMC 018 and 024), and one of the sources with strong crystallines (MSX SMC 134) is classified as 2.SX6.

Figure 5 compares the distribution of the SE indices for the MSX SMC sample (including the 6 objects in Table 3 ) with the LMC sample from Sloan et al. (2008), and the Galactic supergiants and AGB stars from Sloan \& Price (1995, 1998). These comparison samples are also shown in the silicate dust sequence in Figure 4. The MSX sources in the SMC have an overabundance of SE7 and 8 sources in comparison to both the Galactic AGB sample and the LMC sources.

It follows that spectra dominated by alumina are relatively uncommon in the SMC sample compared to the other samples. This difference could arise from metallicity, with the relative lack of aluminum leading to more amorphous silicates and less alumina dust (e.g., Jones et al. 2014). Sloan \& Price (1998) noted that the spectra of Galactic AGB stars are distributed across the silicate sequence, whereas the RSGs are primarily SE5-7 (i.e., the bottom two panels in Figure 5). The SMC sample contains both RSGs and AGB stars, but several of the AGB stars show evidence of hot bottom burning and are likely to be massive (see Section 3.2). At a distance of $60 \mathrm{kpc}$, a bias in our sample for brighter objects would be expected. The often uncertain distances to Galactic AGB stars make it difficult to estimate their luminosity and hence initial mass. However, if the trend favoring amorphous silicates in RSGs carries over to the massive AGB stars, this could explain the lack of alumina dust in the SMC. Distances from the Gaia mission may help address this issue in the Galaxy.

The outlier at $(0.9,1.6)$ in the silicate dust sequence is MSX SMC 134, an SX6 with strong crystalline silicate emission that enhances the $F_{12}$ measurement, and is discussed below (Section 5.2). One other SE6, MSX SMC 018, also has weak crystalline features. None of the evolved stars observed here, or 


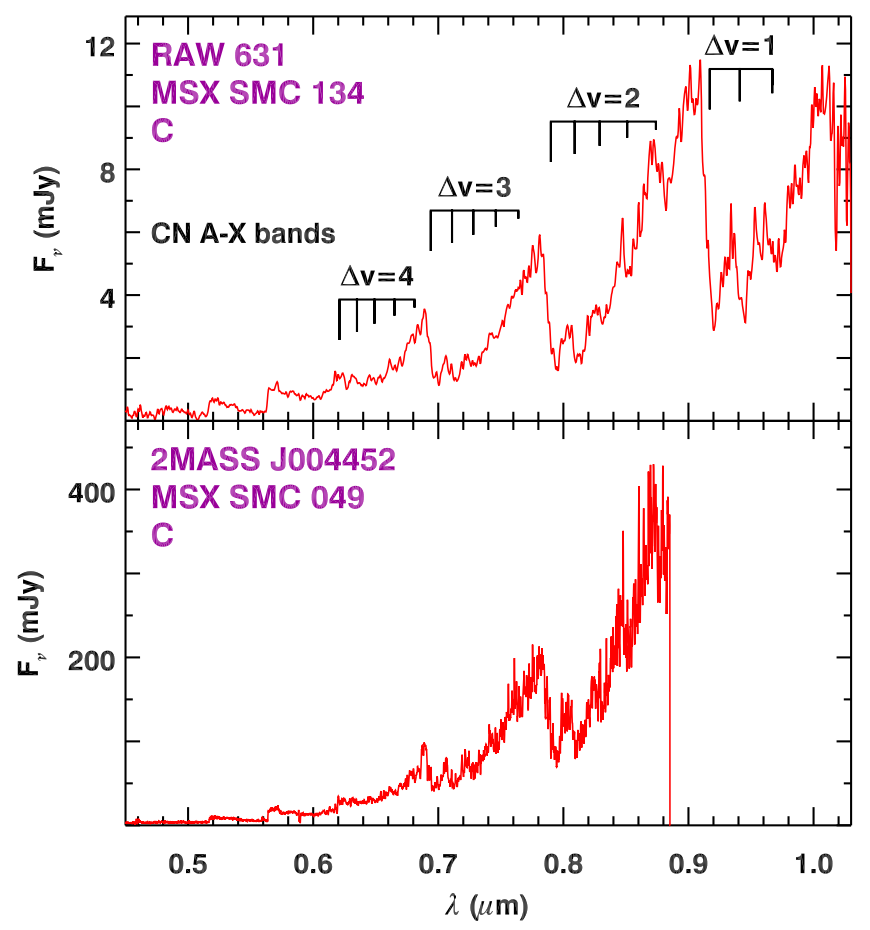

Figure 3. Optical spectra of the evolved stars in program 3277 and 30355 that have carbon-rich optical spectra but oxygen-rich features in their IRS spectra.

in any of the other programs observing the SMC with the IRS (or with any other instrument, to the best of our knowledge), shows silicate features in absorption or self-absorption. In contrast, the LMC contains several sources with optically thick dust, both oxygen-rich (Sloan et al. 2008) and carbon-rich (Gruendl et al. 2008). It is not clear if the lack of optically thick dust in the SMC is a metallicity effect, a bias in the source selection, or some other effect.

With respect to the DEC, we can point out what is a likely selection effect. For the present sample, the DEC ranges from 0.19 to 3.95 , with a mean and median of 1.34 and 0.96 , respectively. As described in Section 2, our sources were selected based on their combined near- and mid-IR colors, with the exception of HV 12122, which has the smallest DEC. The four SMC spectra with silicate emission from Sloan et al. (2008) have DECs of 0.16 to 0.80 , and the 11 naked and nearly naked stars have DECs of 0.0 to 0.24 . Those sources were chosen based on their optical spectra. Not surprisingly, the optically selected sample shows less dust than the infraredselected sample.

\subsection{AGB Versus RSG}

The distinction between an AGB star and an RSG often relies on the bolometric luminosity, with $M_{\text {bol }}=-7.1$ as the classic limit (Paczyński 1971). We derived the bolometric magnitudes for the stars in Tables 2 and 3 using the IRS spectra and the photometry presented in the Appendix. Although most, if not all, of the stars are variables, the luminosity derivation uses multi-epoch photometry, so these values reflect the mean luminosity of the stars. Three of the evolved stars in the tables are near the classic luminosity limit, with $M_{\text {bol }} \sim-7.0$ : HV 12122, MSX SMC 181 (HV 11417), and MSX SMC 210 (HV 12149).

Jones et al. (2012) had calculated $M_{\mathrm{bol}}=-7.1$ for $\mathrm{HV}$ 12122 and called it an RSG. Ruffle et al. (2015), though, classified it as an early-type O-rich AGB star (O-EAGB), a class of long-period variables (LPVs) with weak infrared excess but no distinct dust features. As they noted, $\mathrm{Li}$ absorption has been detected in the optical (Smith et al. 1995), which is an indicator of hot bottom burning (HBB). HBB occurs at the base of the convective envelope in the most massive AGB stars and can push them over the classic $M_{\text {bol }}$ limit (e.g., Bloecker \& Schoenberner 1991; D'Antona \& Mazzitelli 1996; Marigo 1998). Stars like HV 12122, with luminosities near or above the nominal limit and $\mathrm{Li}$ absorption, are mostly likely massive AGB stars and HBB candidates (Smith et al. 1995). In contrast, Smith et al. (1995) did not detect Li in MSX SMC 210, so it may simply be an AGB star relatively near the luminosity limit.

MSX SMC 181 has also been classified as both an AGB and an RSG (Ruffle et al. 2015). Elias et al. (1980) first classified it as an M5e I based on an optical spectrum taken near maximum light, but they also suggest that it could be an unusual type of LPV rather than a real supergiant. The optical spectrum in the bottom-right panel of Figure 2 shows strong TiO absorption bands, similar to the recognized O-AGB stars in our sample (e.g., MSX SMC 018 and 024), as opposed to the spectra of the RSGs (e.g., MSX SMC 096 or 168). Although listed as a semiregular in the GCVS, Soszyński et al. (2011) call it a Mira due to its large pulsation amplitude $(\Delta I=1.86 \mathrm{mag})$. Its luminosity has been estimated to be between $\sim-7.0$ and -7.3 ; our current estimate is -7.02 . Based on its other properties, particularly the optical spectrum and large pulsation amplitude, we call it an O-AGB, with $\mathrm{HBB}$ (sometimes) pushing it above the nominal luminosity limit.

MSX SMC 055 (IRAS 00483-7347) has a luminosity of -7.5, above the nominal limit for AGB stars. Like Hv 12122 and MSX SMC 181, though, it has been categorized as both an RSG and an AGB (Whitelock et al. 1989; Groenewegen \& Blommaert 1998, respectively). Its optical spectrum (Figure 2, see also Groenewegen \& Blommaert 1998) has deep TiO bands similar to the O-AGB star MSX SMC 018, and unlike the RSGs. Again, Soszyński et al. (2011) call it a Mira due to its large pulsation amplitude $(\Delta I=1.73 \mathrm{mag})$ and long period (1860 days). Additionally, Castilho et al. (1998) detected a moderate strength $\mathrm{Li}$ absorption feature at $670 \mathrm{~nm}$ in its optical spectrum. We conclude that this star, too, is a high-mass AGB star where HBB allows it to exceed the classic limit.

\subsection{Hydrocarbons in RSGs}

Along with the typical silicate dust features, three of the unambiguous RSGs in our sample, MSX SMC 096, 101, and 109 , clearly show the $11.3 \mu \mathrm{m} \mathrm{PAH} \mathrm{feature} \mathrm{(Figure} \mathrm{6),} \mathrm{as} \mathrm{was}$ noted by Ruffle et al. (2015) for 096 and 109. None have strong features at the other expected wavelengths of PAHs, although there is a hint at $12.7 \mu \mathrm{m}$ in MSX SMC 109. The other RSGs in Tables 2 and 3 show at most hints of the $11.3 \mu \mathrm{m}$ feature, but are too noisy for clear detections. This feature has previously been observed in RSGs in the Milky Way (Sylvester et al. 1994, 1998; Verhoelst et al. 2009) and the LMC (Sloan et al. (2008), O. C. Jones et al. 2016, in preparation). There is no extended emission apparent in the IRS slit images or in the IRAC images of our three RSGs that would indicate significant contamination in the beam. The emission seems to be localized to the RSG, just as with the other RSGs with PAH emission. 
Table 4

Oxygen-rich Dust Analysis

\begin{tabular}{|c|c|c|c|c|c|c|}
\hline $\begin{array}{l}\text { MSX } \\
\text { SMC }\end{array}$ & {$[7]-[15]$} & $\begin{array}{c}\text { Dust em. } \\
\text { Contrast (DEC) }\end{array}$ & $F_{10} / F_{11}$ & $F_{10} / F_{12}$ & $\begin{array}{c}\text { Corrected } \\
F_{11} / F_{12} \\
\end{array}$ & $\begin{array}{c}\text { SE } \\
\text { Index }\end{array}$ \\
\hline HV 12122 & $0.47 \pm 0.02$ & $0.19 \pm 0.02$ & $1.06 \pm 0.03$ & $1.28 \pm 0.04$ & $1.31 \pm 0.05$ & $\cdots$ \\
\hline 018 & $2.03 \pm 0.00$ & $2.96 \pm 0.01$ & $1.01 \pm 0.01$ & $1.59 \pm 0.06$ & $1.42 \pm 0.05$ & SE6 \\
\hline 024 & $1.40 \pm 0.01$ & $0.96 \pm 0.01$ & $0.95 \pm 0.01$ & $1.27 \pm 0.02$ & $1.29 \pm 0.02$ & SE5 \\
\hline 055 & $1.83 \pm 0.00$ & $2.74 \pm 0.01$ & $1.11 \pm 0.01$ & $1.74 \pm 0.05$ & $1.49 \pm 0.04$ & SE7 \\
\hline 067 & $0.27 \pm 0.01$ & $0.02 \pm 0.01$ & $0.79 \pm 0.17$ & $0.58 \pm 0.13$ & $0.97 \pm 0.30$ & $\ldots$ \\
\hline 096 & $0.62 \pm 0.01$ & $0.23 \pm 0.01$ & $0.72 \pm 0.04$ & $0.87 \pm 0.04$ & $1.08 \pm 0.07$ & SE3 \\
\hline 109 & $0.99 \pm 0.01$ & $0.75 \pm 0.01$ & $1.11 \pm 0.02$ & $1.70 \pm 0.05$ & $1.47 \pm 0.05$ & SE7 \\
\hline 134 & $1.93 \pm 0.01$ & $2.34 \pm 0.03$ & $0.90 \pm 0.01$ & $1.58 \pm 0.07$ & $1.40 \pm 0.06$ & SX6 \\
\hline 149 & $1.09 \pm 0.01$ & $0.90 \pm 0.01$ & $1.21 \pm 0.01$ & $1.67 \pm 0.04$ & $1.47 \pm 0.04$ & SE7 \\
\hline 168 & $0.65 \pm 0.02$ & $0.34 \pm 0.00$ & $1.34 \pm 0.02$ & $2.14 \pm 0.08$ & $1.63 \pm 0.06$ & SE8 \\
\hline 181 & $1.40 \pm 0.01$ & $1.85 \pm 0.01$ & $1.37 \pm 0.03$ & $2.33 \pm 0.07$ & $1.69 \pm 0.06$ & SE8 \\
\hline 101 & $0.87 \pm 0.01$ & $0.37 \pm 0.01$ & $0.82 \pm 0.03$ & $1.03 \pm 0.03$ & $1.18 \pm 0.06$ & SE4 \\
\hline 145 & $1.73 \pm 0.01$ & $2.09 \pm 0.01$ & $1.36 \pm 0.03$ & $2.25 \pm 0.06$ & $1.66 \pm 0.06$ & SE8 \\
\hline 161 & $1.60 \pm 0.02$ & $1.67 \pm 0.01$ & $1.10 \pm 0.02$ & $1.83 \pm 0.05$ & $1.51 \pm 0.05$ & SE7 \\
\hline
\end{tabular}

(This table is available in machine-readable form.)

Sylvester et al. (1998) suggested that the small fraction of RSGs in which they detected PAHs implies that they arise in a short-lived phase with the needed combination of UV flux and outflow chemistry, a combination that is not present in AGB stars. Another possibility is that the phenomenon is similar to the Pleiades effect, with the RSGs embedded in diffuse material, since Li \& Draine (2002) found that optical photons are sufficient to excite (pre-existing) PAHs. This idea, however, requires that the material containing the PAHs seen in the original RSGs in $h$ and $\chi$ Per must be quite patchy within the clusters, as not all of the RSGs in those clusters show the feature.

\section{THE NATURE OF THE INFRARED-BRIGHT STELLAR POPULATION IN THE SMC}

\subsection{Anticipated Object Types}

One of the goals of this project was to test the selection criteria used to build the source sample. As mentioned in Section 2.1, the targets were selected based on their $J-K_{s}$ and $K_{s}-A$ colors, supplemented by $H-K_{s}$. Egan et al. (2001) had found that color space distinguished among different populations of evolved stars in the LMC. They used the SKY model of Wainscoat et al. (1992) combined with the known object types for $\sim 1000$ objects in the LMC. As the SKY model was developed from data on Galactic objects, yet seemed to work well in the more metal-poor environment of the LMC, it was expected that the SMC would show a similar agreement.

Figure 7 compares the expected types of MSX SMC objects based on their MSX-2MASS colors (left-hand panel) and the actual types based on their IRS spectra (right-hand panel). The figure includes the SMC sources in the MSX catalog that were observed by other programs, as well as our own. As can be seen, more carbon-rich objects were observed than originally anticipated. About one-third of the proposed sample was expected to be carbon rich, and almost half were expected to be $\mathrm{OH} / \mathrm{IR}$ stars. Instead, two-thirds were carbon rich and no $\mathrm{OH} /$ IR stars were detected. The reddest oxygen-rich objects are crystalline silicate sources, rather than $\mathrm{OH} / \mathrm{IR}$ stars.

Zijlstra et al. (2006) also found this overabundance of carbon-rich objects in their IRS observations in the LMC. They suggested that the models did not well represent the objects with high mass-loss rates, i.e., the redder objects. Furthermore, the oxygen-rich and carbon-rich sources overlap more in these colors than the SKY model would predict. The carbon stars form a well-defined sequence, but the oxygen-rich stars are more scattered. So, if one is selecting sources near the carbon star locus, one is likely to get a carbon-rich source, even when there are also oxygen-rich objects with similar colors.

\subsection{James Webb Space Telescope (JWST) Color-Color Diagrams}

Several groups have used the modeled and observed photometry from IRAC, often combined with near-infrared and optical data, to create color-magnitude and color-color diagrams that distinguish among types of objects such as YSOs, carbon stars, O-rich AGBs, etc. (e.g., Whitney et al. 2003; Bolatto et al. 2007; Boyer et al. 2011, to name just a few). Rapidly approaching, however, is the upcoming launch of the JWST, which will host instruments with a different set of filters in the near- and mid-infrared. The MidInfrared Instrument (MIRI; Rieke et al. 2015), in particular, includes a set of nine filters that span the wavelength range from 5 to $28 \mu \mathrm{m}$ (Bouchet et al. 2015), an excellent match to the IRS range of 5-35 $\mu \mathrm{m}$.

Although JWST will be more sensitive than Spitzer, it will typically be observing more distant and fainter galaxies. The individual sources that will be detected will be the brightest, most luminous objects, similar to the point sources detected in the SMC by MSX. We can gain insight into what JWST may see by comparing the MIRI filters to the IRS data. We have convolved the MIRI filter functions with the 59 IRS spectra of the MSX SMC sources and considered several combinations for color-color diagrams. Because the MIRI 5.6 $\mu \mathrm{m}$ filter extends slightly blueward of the IRS spectra, we extrapolate to the IRAC [4.5] photometry. This shifts the colors slightly, but makes no qualitative difference to the color-color diagrams here. Figure 8 shows four sets with good separation of one or more specific object types given in Tables 2 and 3 .

Obviously, this will not be the last word on color-color diagrams for JWST (see, e.g., Jones et al. 2016). A few conclusions can be drawn, though, from the MIRI-based diagrams. The best filters we found for separating the carbon stars from the O-rich AGB and RSG stars were the 5.6, 7.7, and 


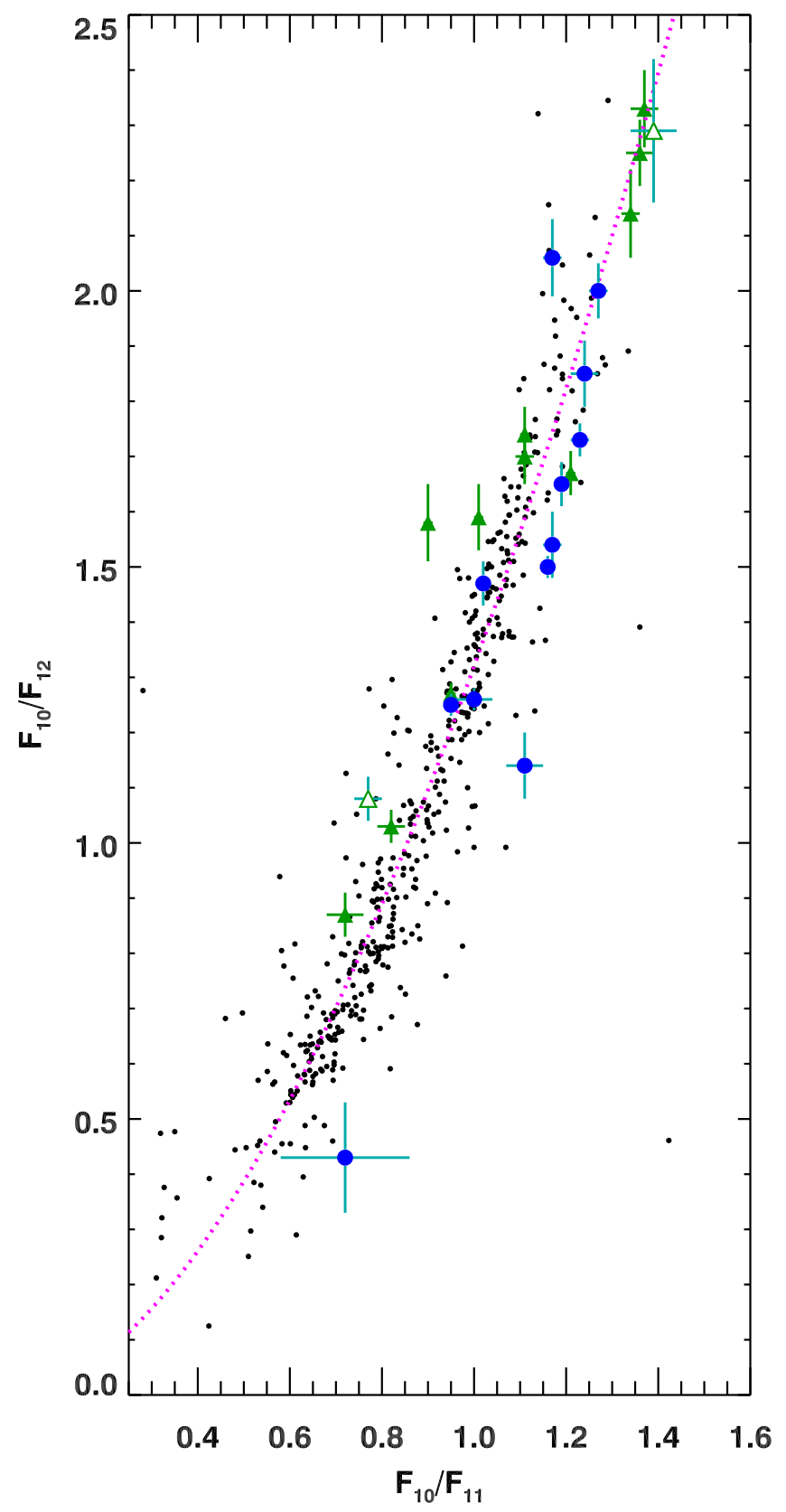

Figure 4. Silicate dust sequence. The green filled triangles are the SMC sources from this work. The small black dots are Galactic AGB sources from Sloan \& Price $(1995,1998)$, the blue filled circles and green open triangles are the LMC and SMC sources from Sloan et al. (2008), respectively. The dotted line is the power-law fit by Sloan et al. (2008).

$21.0 \mu \mathrm{m}$ filters (upper-left panel). Most of the other combinations had more overlap between these types of evolved stars.

Many combinations can be used to distinguish the YSOs from the evolved stars, as the YSOs tend to be redder in at least one color. The YSOs in the bottom two panels separate into two groups. The five bluer sources all show silicate absorption (SA) features, and in the Hanscom system (Kraemer et al. 2002b), would be classified as 4 or 5.SAi, with the "i" indicating ice absorption. The most prominent spectral feature for the redder four sources (one of which is an $\mathrm{H}$ II region, not a YSO) is PAH emission, along with fine-structure lines, and these would be classified together as 5.UE (for UIRs + emission lines). In contrast, the SAGE-Spec classifications

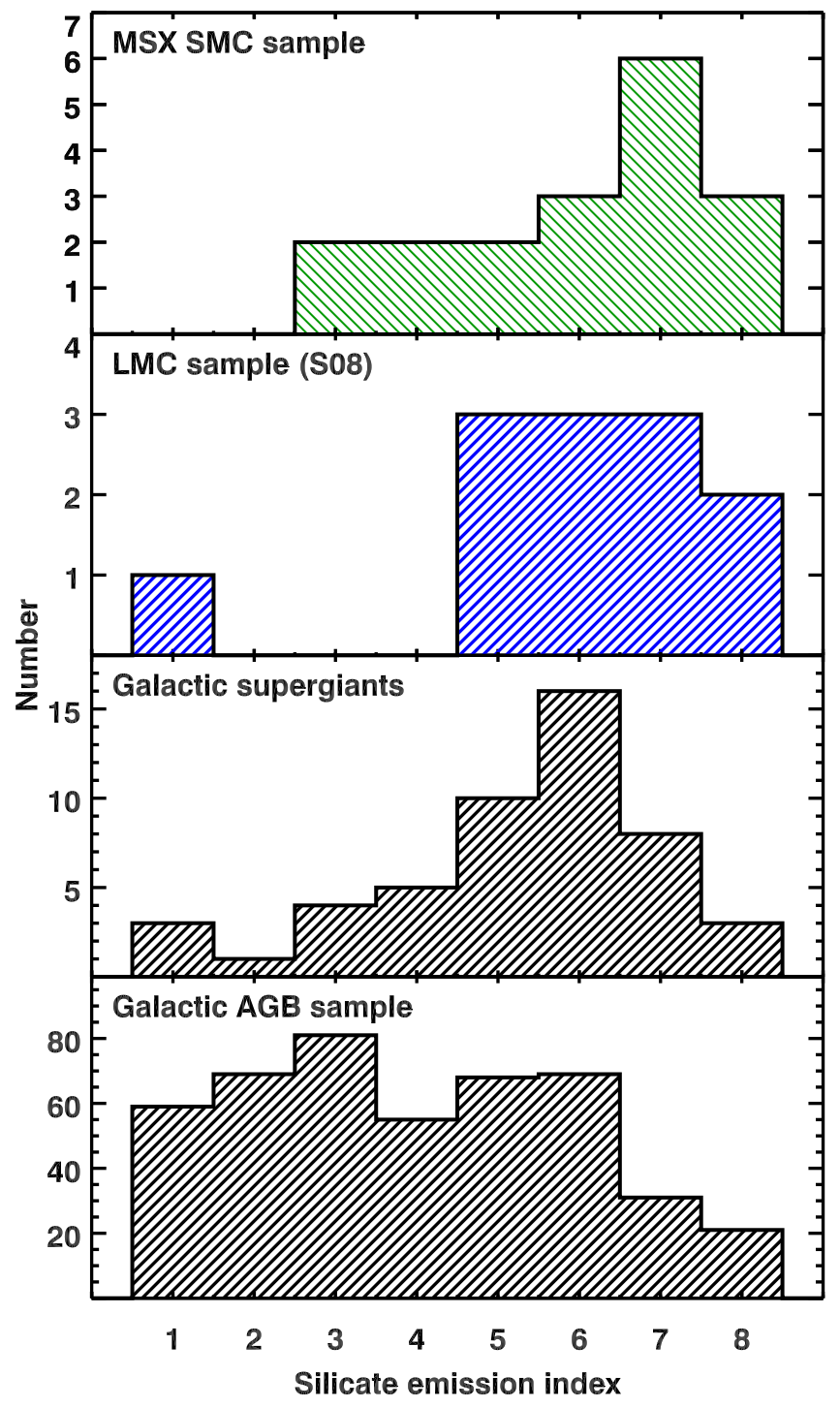

Figure 5. Distribution of the silicate emission index for, from top to bottom: the SMC MSX sample, the LMC sample (Sloan et al. 2008), the Galactic supergiants, and the Galactic AGB stars (Sloan \& Price 1995, 1998).

(Woods et al. 2011; Ruffle et al. 2015) classed two of the redder sources as YSO-1s, since an early distinguishing feature in that decision tree is ice absorption rather than silicate absorption; one of the bluer sources is classed as a YSO-2, i.e., no ices.

We did not, of course, look at all 154 three- and four-filter combinations, let alone all those that could be generated with shorter wavelength filters from the Near-IR Camera (NIRCam; Beichman et al. 2012). Other filter combinations will undoubtedly highlight other categories of objects, including different SAGE-Spec YSO types. The [21]-[25] color, for example, could potentially be used to find $\mathrm{MgS}$ or $21 \mu \mathrm{m}$ features in carbon stars.

\section{NOTES ON INDIVIDUAL SOURCES}

\subsection{HV 12122 and MSX SMC 067}

HV 12122 is the source observed in program 3277 too faint to appear in the MSX SMC catalog. It was selected to fill out the samples of blue stellar sources expected to be relatively dust free, and indeed it has one of the infrared spectra with no 


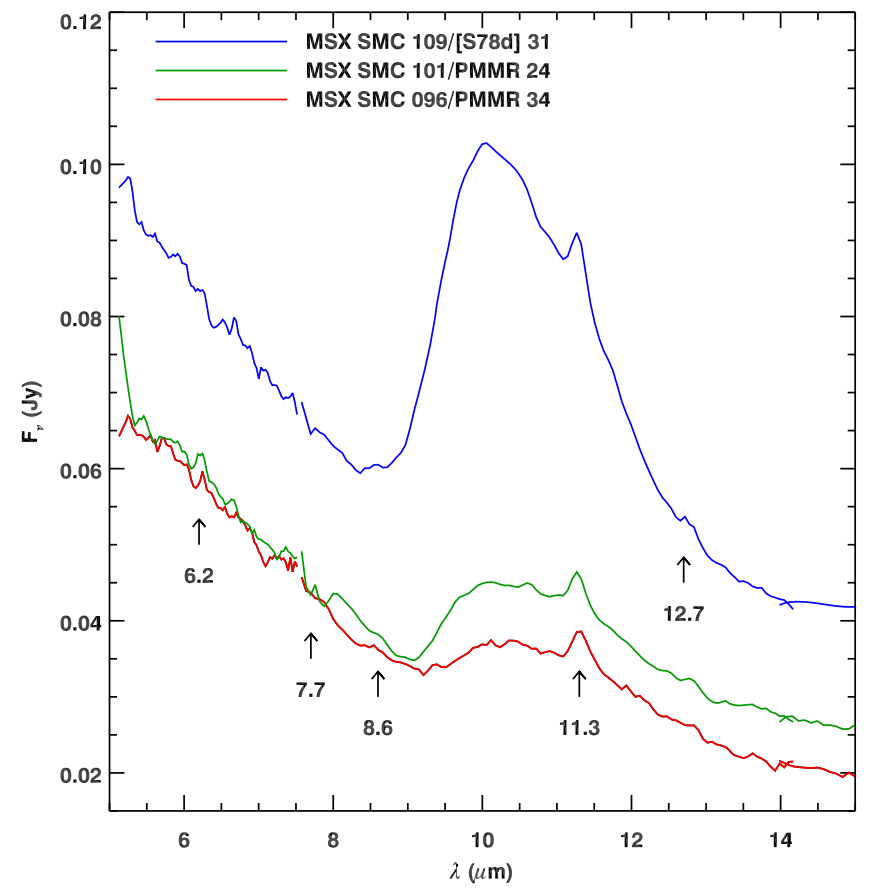

Figure 6. Three RSGs with clear $11.3 \mu \mathrm{m}$ PAH emission. The $12.7 \mu \mathrm{m}$ feature is tentatively detected in MSX SMC 109, but none of the other features are convincing in it or the other RSGs.

obvious dust features. MSX SMC 067, aka HV 11262, also has a featureless IRS spectrum. However, the spectra both fall less steeply than Rayleigh-Jeans tails would, and they have measurable excesses, both in [7]-[15] color and in DEC.

The $\mathrm{S} / \mathrm{N}$ in the dust excess is too low to allow a meaningful SE classification, in both HV 12122 and MSX SMC 067. What we have for each is a generally featureless low-contrast excess, which could conceivably be fitted with amorphous aluminarich dust. Another possible carrier is iron-rich dust, as McDonald et al. (2010) proposed to explain low-contrast featureless excesses in low-mass oxygen-rich AGB stars observed in globular clusters.

We classified HV 12122 as O-EAGB based on its optical spectrum (Figure 2), which clearly shows the absorption band structure characteristic of $\mathrm{TiO}$. The remainder of the stars classified here as O-AGB were identified based on silicate dust emission features in their infrared spectra. MSX SMC 067 was classified by R15 as an RSG due to its optical spectral type, which is supported by the bolometric magnitude that we calculate, $M_{\text {bol }} \sim-7.9$.

\subsection{MSX SMC 134}

The infrared spectrum of MSX SMC 134 shows prominent emission features from crystalline silicates at 19.5, 23.5, 28, and $33.5 \mu \mathrm{m}$, which are among the strongest that have been seen in AGB and RSG stars (Jones et al. 2012). Even more intriguing, the 2MASS point source at this location is associated with RAW 631, a carbon star (Rebeirot et al. 1993; Raimondo et al. 2005).

Despite some extended emission in the region, the positions of the IRAC source, MIPS source, and 2MASS source are all within 0 ". 5 of each other. Synthetic photometry from our IRS spectrum agrees with the IRAC $8 \mu \mathrm{m}$ photometry of MSX SMC 134 within $0.26 \mathrm{mag}$ and with the MIPS $24 \mu \mathrm{m}$ photometry within 0.05 mag. We have carefully examined the area around RAW 631 to ensure that the optical and infrared spectrum are of the same source. Another source lies $\sim 6^{\prime \prime}$ to the northwest in the 2MASS images and in the Epoch 1 SAGESMC catalog, but it is $1 \mathrm{mag}$ fainter at $J$ and 4 mag fainter at $8 \mu \mathrm{m}$. No other objects appear within $10^{\prime \prime}$, and we conclude the IRS spectrum is of this source.

The optical spectrum (Figure 3) confirms the carbon-rich nature of the star, with strong absorption from the A-X CN bands throughout the optical region. Van Loon et al. (2008) obtained 3-4 $\mu \mathrm{m}$ spectra for a large set of IRS-targeted SMC stars, including MSX SMC 134. Its spectrum shows some of the weakest acetylene $\left(\mathrm{C}_{2} \mathrm{H}_{2}\right)$ and hydrogen cyanide $(\mathrm{HCN})$ absorption features in their $\mathrm{C}$-rich sample (their Figures 3 and 10). The $3.1 \mu \mathrm{m}$ band is clearly detected, but the other features are not readily apparent in the spectrum. In contrast, the midinfrared spectrum is dominated by silicate dust, with no hint of carbon-rich chemistry. Thus, the 3-4 $\mu \mathrm{m}$ region examined by van Loon et al. (2008) is a transition region between the C-rich optical emission and the silicate dust seen in the IRS spectrum.

Jones et al. (2012), who investigated crystalline silicates in the Milky Way and Magellanic Clouds, suggested that MSX SMC 134 is a silicate/carbon star. MSX SMC 134 would be the first silicate/carbon star detected outside the Milky Way. ${ }^{13}$ The current hypothesis is that the oxygen-rich dust, a remnant of previous mass loss while the star was oxygen-rich, is stored in a disk around a binary system, where it could survive long enough for detection (Lloyd Evans 1990, 1995), and this remains the most likely explanation (Yamamura et al. 2000; Lloyd Evans 2010). The strong crystalline silicate features in the spectrum are also consistent with the notion of a disk (e.g., Molster et al. 1999; Gielen et al. 2008, 2011). Dust grains in a disk will remain warm, and they can be photo-processed, annealing the grains from an amorphous to a more crystalline structure.

MSX SMC 134 stands out among the previously known silicate/carbon stars in two ways. First, most show little or no variability (e.g., Lloyd Evans 1990; Kwon \& Suh 2014). The MACHO observations of MSX SMC 134, though, show a clear variability (Figure 9) with two periods of $\sim 1260$ and 144 days. These are in good agreement with previous investigations using MACHO or OGLE data, which found periods of 1255 and 145 days (Raimondo et al. 2005), or 141 days (Soszynski et al. 2011), respectively.

Second, its crystalline silicates are in contrast with the more usual amorphous silicate features at 9.7 and $18 \mu \mathrm{m}$ seen in most other silicate/carbon stars. There are three others that show crystalline structure: the two post-AGB objects HD 44179 (the Red Rectangle) and EP Lyr, as well as IRAS 09425-6040. The latter has sometimes been considered as a post-AGB star but is in the "disqualified" list of (i.e., Szczerba et al. 2007, stars that have M spectral types or are C-AGB stars). MSX SMC 134 may be more like these objects than the typical silicate/carbon star (although with only a few dozen known, the whole category is rare).

\footnotetext{
13 Trams et al. (1999) proposed that IRAS 04496-6958 in the LMC might be a silicate/carbon star, based on its spectrum from the ISOCAM CVF on board the Infrared Space Observatory. It is certainly carbon rich, but its infrared spectrum does not show silicate emission (Speck et al. 2006). Rather, it has some of the deepest acetylene absorption ever detected in the $12-16 \mu \mathrm{m}$ region, and this, along with the cutoff wavelength of the CVF, conspired to mislead them.
} 


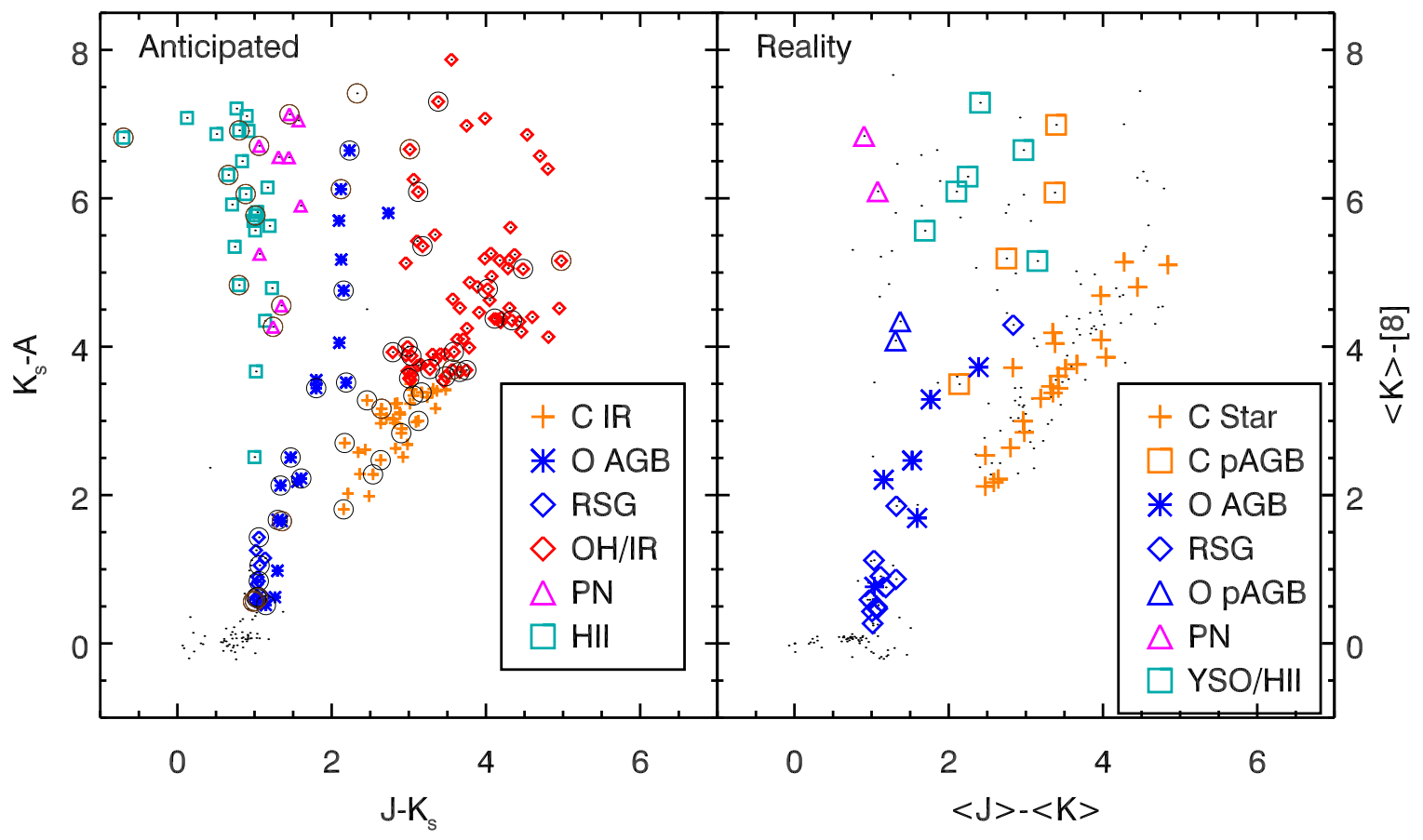

Figure 7. (Left) Anticipated distribution of object types in the MSX SMC catalog from their MSX-2MASS colors. Circles indicate the sources that were observed with the IRS. The clumps of dots at $K_{s}-A \sim 0$ and $J-K_{s} \sim 0-1$ are main sequence stars, and the small number of dots with redder colors are unclassified. (Right) The actual object types as determined by their IRS spectra. The near-infrared colors are from the mean photometry given in the Appendix, and the [8] data are from IRAC Band 4. The dots here are the MSX SMC sources without IRS spectra.

\subsection{MSX SMC 049}

Ruffle et al. (2015) classified MSX SMC 049 as an O-AGB star based primarily on the crystalline silicate features in the $20-35 \mu \mathrm{m}$ range of its IRS spectrum. However, it also has a very deep acetylene absorption feature (Q branch) at $13.7 \mu \mathrm{m}$ and a possible (weak) silicon carbide emission feature. In the optical, Raimondo et al. (2005) and Soszyński et al. (2011) photometrically classified it as a carbon star. Our optical spectrum confirms the carbon-rich chemistry of the source (Figure 3).

The overall shape of the IRS spectrum of this mixed chemistry source is reminiscent of the Magellanic post-AGB stars SMP LMC 11 (Bernard-Salas et al. 2006) and MSX SMC 029 (Kraemer et al. 2006), shown in Figure 10. The more complex hydrocarbons that were detected in those objects (e.g., polyacetelynes and $\mathrm{HC}_{x} \mathrm{~N}$ in SMP LMC 11, PAHs and (tentatively) polyacetelynes in MSX SMC 029) are not as readily apparent in MSX SMC 049, but there is a hint of absorption at $15 \mu \mathrm{m}$ that could be the $\mathrm{C}_{6} \mathrm{H}_{6}+\mathrm{HC}_{3} \mathrm{~N}$ feature. The shoulder around $14 \mu \mathrm{m}$ could be $\mathrm{HCN}$ absorption, and the broad absorption blueward of 13.7 might be the $\mathrm{C}_{2} \mathrm{H}_{2} \mathrm{R}$ branch.

We extracted the $\mathrm{C}_{2} \mathrm{H}_{2}$ at 7.5 and $13.7 \mu \mathrm{m}$, as well as the [6.4]-[9.3] color and silicon carbide features using the same wavelength ranges as Sloan et al. (2006b). The equivalent width of the $7.5 \mu \mathrm{m}$ feature is relatively weak, and the $13.7 \mu \mathrm{m}$ feature is not as strong as anticipated from the spectrum. The [6.4]-[9.3] color, though, is as red as expected, and the silicon carbide strength is similar to the weak $\mathrm{SiC}$ sources of Sloan et al. (2006b, their Table 5). Given the apparent strength of the $13.7 \mu \mathrm{m}$ feature in the spectrum, which probably contains the $\mathrm{R}$ branch, a more complex treatment of these features may be needed to correctly assess the carbon-rich features.
For the crystalline features, the feature-to-continuum ratios were fit following the methods used by Jones et al. (2012). Except for the $19 \mu \mathrm{m}$ feature, these are unlikely to be effected by any of the carbon-rich features at the shorter wavelengths. In the context of the large sample of crystalline silicate sources considered by Jones et al., the 28 and $33 \mu \mathrm{m}$ features of MSX SMC 049 are among the five strongest feature-to-continuum ratios, with only their two SMC disk candidates (one of which is MSX SMC 134) being stronger.

As with the two carbon-rich post-AGB candidates shown in Figure 10, the dust continuum is not well fit with a single temperature, but has contributions from dust at $T_{d} \sim 320-550$ K. We use the models of Sargent et al. (2011) to fit the infrared spectral energy distribution and estimate the dust mass-loss rate for MSX SMC 049 to be $\dot{M}_{d} \sim 4.5 \times 10^{-8} M_{\odot} \mathrm{yr}^{-1}$, although these are not ideal for mixed chemistry sources. The models also suggest $L \sim 6.6 \times 10^{4} \quad L_{\odot}, \quad T_{\text {eff }} \sim 3800 \mathrm{~K}$, and $R_{*} \sim 370 R_{\odot}$.

\subsection{MSX SMC 145}

At first glance, the IRS spectrum of MSX SMC 145, aka BMB-B 75, appears to be a fairly normal silicate emission spectrum contaminated by a cooler dust continuum rising toward the red and a few emission lines. Closer inspection of the location of the narrower emission features, however, shows that they are not at the wavelengths of any known molecular or fine-structure lines. Instead, they appear to be redshifted emission from a background galaxy that aligns perfectly with our intended target. The two features at 13.4 and $15.3 \mu \mathrm{m}$ correspond to the PAH feature at $11.3 \mu \mathrm{m}$ and the [Ne II] line at $12.8 \mu \mathrm{m}$ at a redshift of $z \sim 0.16$. It then follows that the feature at $18.5 \mu \mathrm{m}$ is the $15.5 \mu \mathrm{m}$ [Ne III] line, and the 6.3 $\mu \mathrm{m}$ PAH feature is tentatively detected at $7.5 \mu \mathrm{m}$. Figure 11 

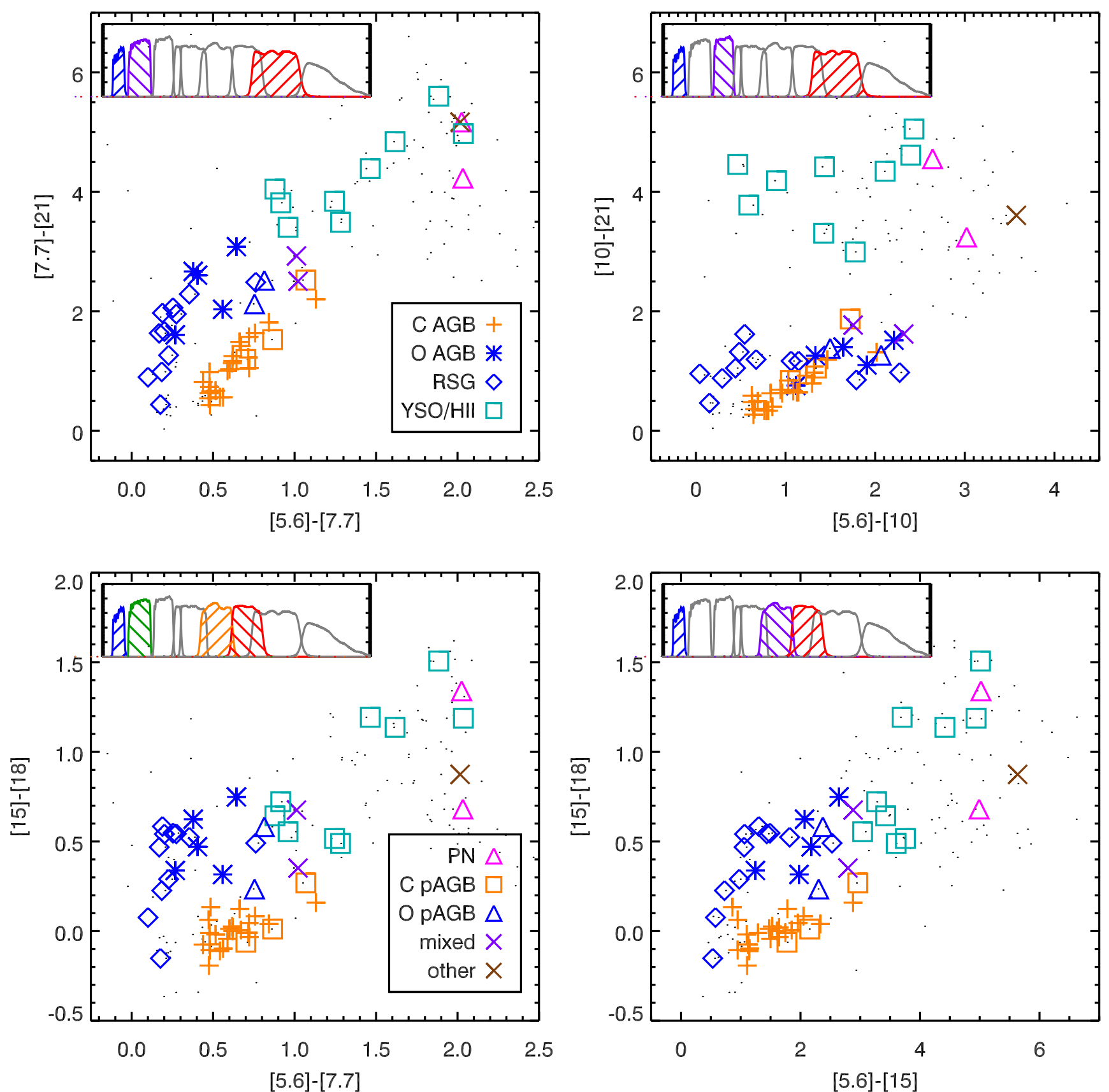

Figure 8. Color-color diagrams generated from the IRS spectra using MIRI filter functions. The colored symbols are the MSX sources, with the IRS-based object types given in the legends; the black dots are the remaining SMC sources with IRS spectra. (The source marked "Other" is MSX SMC 153, a B[e] star.) As the MIRI filters are not yet very familiar, we include them in the upper left of each panel, with the colored hatching indicating which filters are used in each plot.

shows the 5-20 $\mu \mathrm{m}$ data with the redshifted emission features labeled. Polsdofer et al. (2015) suggested that this object might be the first $\mathrm{OH} / \mathrm{IR}$ star found in the SMC, as it is the only star in their sample with a broadband detection at $6 \mathrm{~cm}$ (Wong et al. 2012), which could potentially be from $\mathrm{OH}$ masers. Jones et al. (2015) note that MSX SMC 145 is the only AGB star in the SMC with far-infrared emission detected by Herschel (only one or two O-AGB stars in the LMC were detected, as well). Based on the IRS spectrum and the redshifted fine-structure lines, it seems more likely the radio and far-infrared emission are from the background galaxy, so that there are still no known OH/IR stars in the SMC (e.g., van Loon 2012).

\section{SUMMARY}

We have observed a set of infrared-bright MSX sources in the SMC with Spitzer's IRS and analyzed those spectra with oxygen-rich dust features. There are relatively more sources with an index of SE7 and SE8 on the silicate sequence (Sloan \& Price 1995, 1998) compared to LMC or Milky Way samples. This could be due to the number of RSGs in our sample or caused by a metallicity-induced difference in the dust composition in the SMC. Three of the sources with luminosities near or above the classic AGB/RSG boundary are likely still on the AGB, but with luminosities elevated due to HBB. 

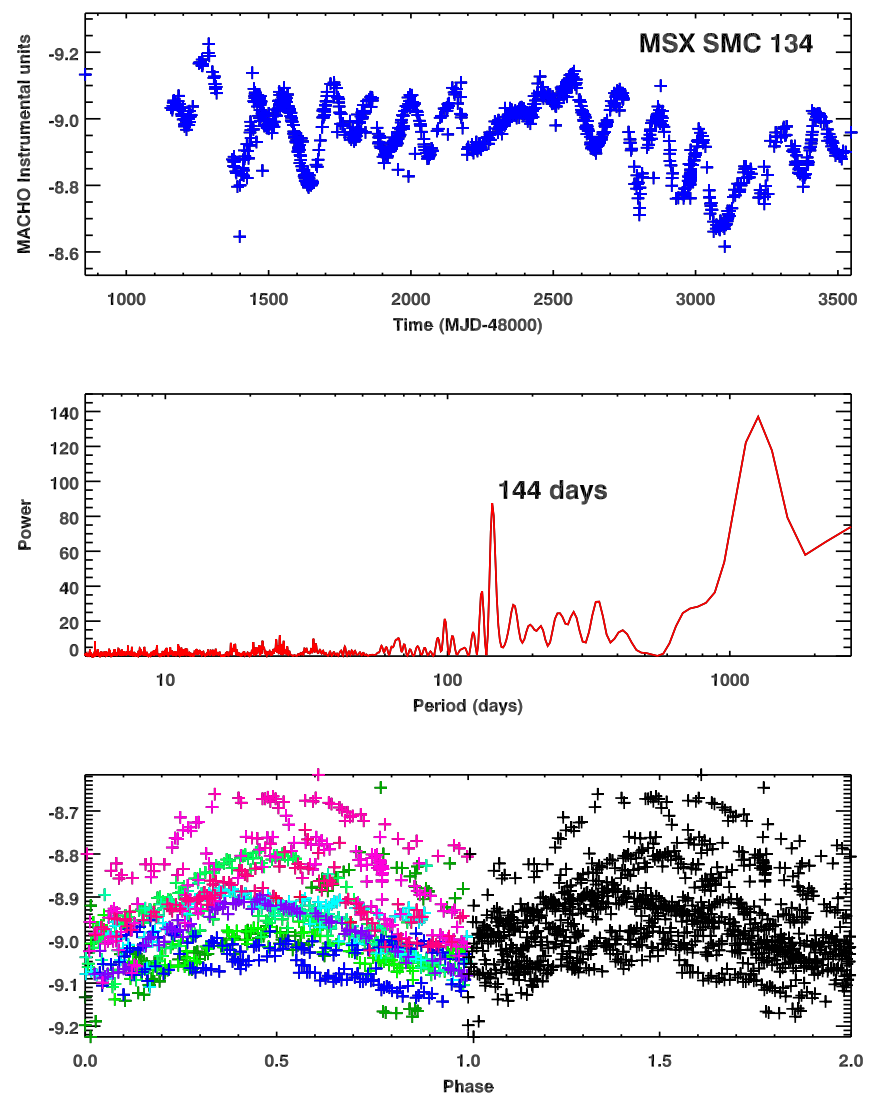

Figure 9. (Top) Red-band MACHO data for MSX SMC 134. (Middle) Periodogram results, showing a strong peak at 144 days. (Bottom) Data folded about the 144 day period; two phases are shown for clarity. The colors show sets of 144 days of data.

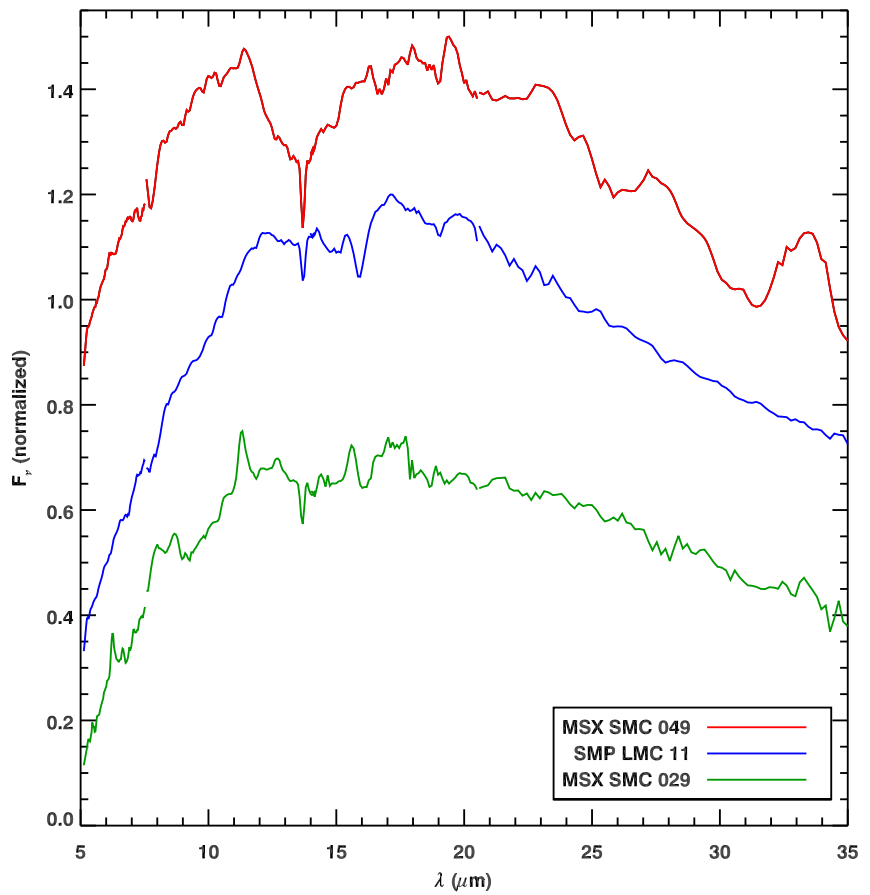

Figure 10. Comparison of MSX SMC 049 (red) with SMP LMC 11 (blue) and MSX SMC 029 (green).

The infrared-based selection criteria are likely responsible for the larger average DEC in our sample compared to the SMC sources of Sloan et al. (2008), who chose their sources based on

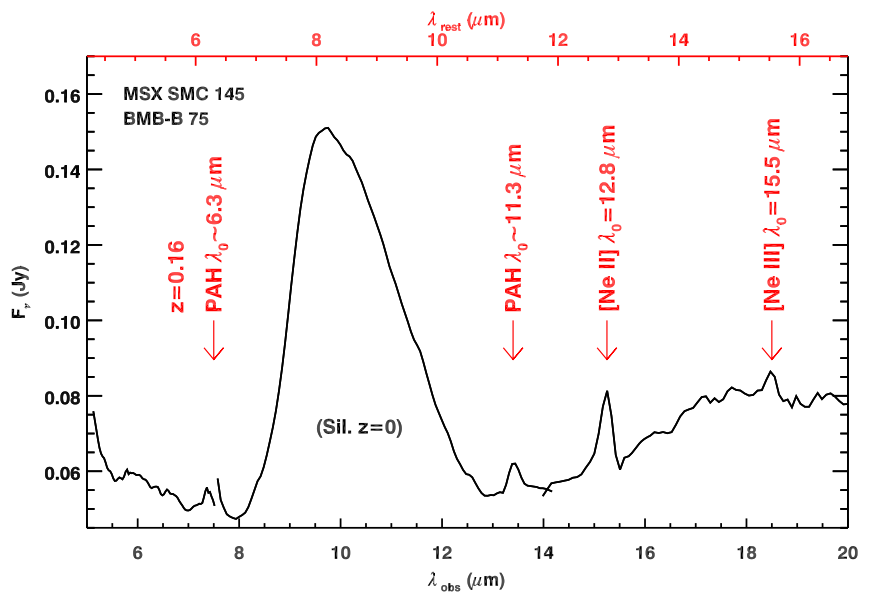

Figure 11. Redshifted fine-structure lines in the spectrum of MSX SMC 145. The $9.7 \mu \mathrm{m}$ and $18 \mu \mathrm{m}$ silicate features belong to the O-rich AGB star in the SMC that was the intended target.

optical spectra. No deeply embedded evolved stars of either oxygen-rich or carbon-rich chemistry have been found in the $\mathrm{SMC}$, in contrast to the populations of $\mathrm{OH} / \mathrm{IR}$ and extreme carbon stars seen in the LMC and Milky Way. The reddest oxygen-rich sources in the SMC are those with crystalline silicate features. The discrepancies between the expected source types and those observed is likely due to how older models treat sources with high mass-loss rates, as well as the overabundance of carbon stars due to the lower metallicity in the SMC.

Over half of the oxygen-rich sources in our sample have unusual spectral features, as opposed to the LMC and Milky Way samples of silicate dust sources from IRAS or ISO, which are dominated by ordinary SE sources with no additional features. Four sources have crystalline silicate features, including two that are among the strongest observed by the IRS in both the LMC and SMC.

Several sources show a mixed chemistry, with both oxygenrich and carbon-rich features in their optical or IRS spectra. Three of the RSGs have the $11.3 \mu \mathrm{m}$ PAH feature, but no other $\mathrm{PAH}$ emission in their spectra. MSX SMC 134 is the first silicate/carbon star detected in the SMC, a carbon star at optical wavelengths but with strong crystalline silicate features in the IRS range. MSX SMC 049, which is also an optical carbon star, has both carbon-rich molecular absorption and strong crystalline silicate features in its IRS spectrum and may be a post-AGB object. Lastly, MSX SMC 145, which was suggested as the first $\mathrm{OH} / \mathrm{IR}$ star in the SMC, is actually an AGB star whose IRS spectrum is contaminated by a background galaxy at a redshift of $z \sim 0.16$.

K.E.K. and M.P.E. were supported in part by NASA via the Air Force Research Laboratory. Support for G.C.S. was provided by NASA through contract No. 1257184 issued by the Jet Propulsion Laboratory (JPL), California Institute of Technology under NASA contract 1407. O.C.J. acknowledges support from NASA grant NNX14AN06G. This research has made use of NASA's Astrophysical Data System Bibliographic Services, the Simbad and VizieR databases operated at the Centre de Données astronomiques de Strasbourg, and the Infrared Science Archive at the Infrared Processing and Analysis Center, which is operated by JPL. We thank the JWST Helpdesk and the MIRI team for making the MIRI filter 

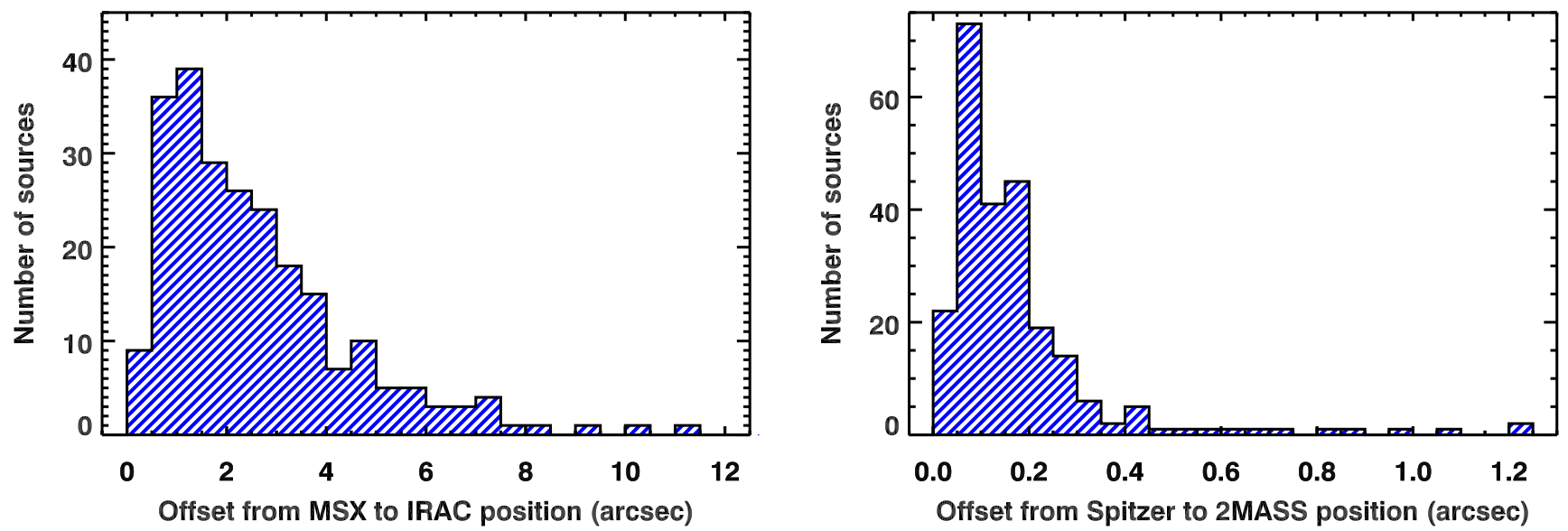

Figure 12. (Left) Distributions of offsets between the MSX and Spitzer positions of the sources in the MSX SMC Catalog. (Right) Distributions of offsets between the new Spitzer-based positions and the associated 2MASS 6x source.

Table 5

MSX SMC Source Positions

\begin{tabular}{|c|c|c|c|c|c|c|c|c|c|}
\hline $\begin{array}{l}\text { MSX } \\
\text { SMC }\end{array}$ & $\begin{array}{l}\text { Alternate } \\
\text { Name }\end{array}$ & \multicolumn{2}{|c|}{$(\mathrm{J} 2000)$} & $\begin{array}{l}\text { Pos'n } \\
\text { References }\end{array}$ & $\begin{array}{l}\text { Morph. } \\
\text { Grade }\end{array}$ & $\begin{array}{l}\mathrm{M}_{\mathrm{bol}} \\
(\mathrm{mag})\end{array}$ & $\begin{array}{l}\text { Obj. } \\
\text { Type }\end{array}$ & $\begin{array}{l}\text { Type } \\
\text { References }\end{array}$ & Notes \\
\hline 100 & LHA $115-\mathrm{N} 29$ & 12.152192 & -72.966919 & 2MASS & B & -5.80 & $\mathrm{PN}$ & BS09 & $\cdots$ \\
\hline 101 & PMMR 24 & 12.215924 & -73.377739 & 2MASS & A & -7.61 & RSG & $\mathrm{R} 15, \mathrm{~K} 16$ & $\cdots$ \\
\hline 103 & HD 5302 & 13.276275 & -73.109299 & 2MASS & A & $\cdots$ & $\cdots$ & $\cdots$ & FG \\
\hline 104 & 2MASS J00540342-7319384 & 13.514259 & -73.327339 & 2MASS & A & -6.06 & YSO & $\mathrm{O} 13$ & $\cdots$ \\
\hline 105 & OGLE SMC-LPV-5091 & 11.258941 & -72.873428 & 2MASS & A & -5.06 & C-AGB & S06 & $\cdots$ \\
\hline
\end{tabular}

Note. FG: probable foreground star; CL: probable cluster member. The sources shown here start with MSX SMC 100 as the first several objects have nothing in the last four columns.

${ }^{a}$ Object Type References: BS09: Bernard-Salas et al. (2009), J12: Jones et al. (2012), K05: Kraemer et al. (2005), K06: Kraemer et al. (2006), K16: this work, L07: Lagadec et al. (2007), O13: Oliveira et al. (2013), R15: Ruffle et al. (2015), S06: Sloan et al. (2006b), S08: Sloan et al. (2008), S14: Sloan et al. (2014), S16: Sloan et al. (2016).

(This table is available in its entirety in machine-readable form.)

functions available and the referee for helpful suggestions to clarify the paper.

\section{APPENDIX \\ CATALOG OF MSX SOURCES IN THE SMC}

This Appendix presents a catalog based on the 243 sources detected in the MSX survey of the SMC, updated with positions and photometry from more recent space-based missions and ground-based surveys. MSX mapped the infrared sky from 1996 to 1997, concentrating on the Galactic Plane (Price et al. 2001) and specific regions such as the LMC (Egan et al. 2001) and SMC. While subsequent surveys of the SMC with Spitzer have achieved higher spatial resolution and sensitivity, the MSX catalog of the SMC remains a useful survey for multiple reasons. First, it provides a flux-limited snapshot of the brightest objects in the SMC. Second, it makes a good comparison with the MSX catalog of the more metalrich LMC (Egan et al. 2001). And finally, it has served as the basis for a number of follow-up projects, including the spectroscopic Spitzer survey described in this paper.

The MSX SMC Catalog is a subset of the high-latitude portion of the MSX Catalog, Version 2.3 (Egan et al. 2003). ${ }^{14}$

$\overline{14}$ Available on VizieR or via Aladin as catalog V/114.
The high-latitude catalog contains 10,168 entries with $|b|>6^{\circ}$, and of these, 243 lie in the range with $7^{\circ} .0<$ R.A. $<18.7$ and $-74.4<$ Decl. $<-71.5$. The MSX SMC Catalog consists of these 243 sources, numbered sequentially from 1 to 243 , with corresponding entry numbers in the high-latitude catalog of 9918 to 10160. All 243 sources are detected in MSX Band A, but the majority have no valid detections in the other MSX bands.

\section{A.1. Updated Positions}

The revisions to the MSX SMC positions are based on midIR observations with Spitzer and the Wide-field Infrared Survey Experiment (WISE; Wright et al. 2010), near-IR data from 2MASS (Cutri et al. 2003; Skrutskie et al. 2006), the Deep Near-IR Survey of the Southern Sky (DENIS; Cioni et al. 2000), and the IR Survey Facility (IRSF; Kato et al. 2007), and optical data from the Magellanic Clouds Photometric Survey (MCPS; Zaritsky et al. 2002) and the OGLE-III catalog of LPVs in the SMC (Soszyński et al. 2011). ${ }^{15}$ Most of the catalogs are available at the Infrared Science Archive at Caltech. The exceptions are the IRSF and

\footnotetext{
15 OGLE is the Optical Gravitional Lensing Experiment, and their catalogs are at ogledb.astrouw.edu.pl/õgle/CVS/.
} 


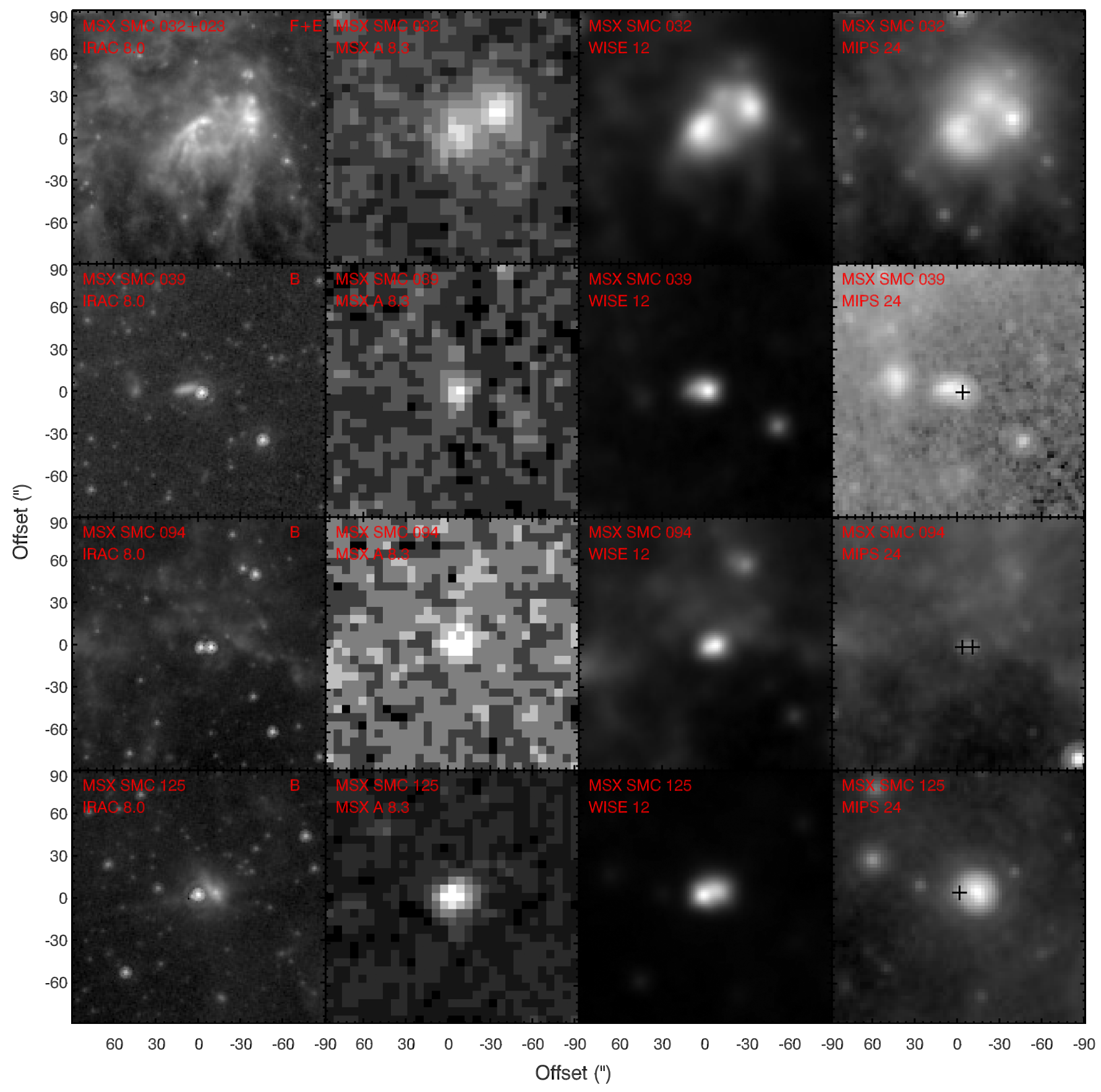

Figure 13. Comparison of four MSX SMC sources with non-A grades. The top row shows MSX SMC 032 and 023 on the left and right, grades F and E, respectively. The lower rows show the three grade B sources described in the text. From left to right, the panels are IRAC $8 \mu \mathrm{m}$, MSX $8.3 \mu \mathrm{m}$, WISE $12 \mu \mathrm{m}$, and MIPS $24 \mu \mathrm{m}$. The black plus marks in the MIPS panel show the locations of any 2MASS point sources within $3^{\prime \prime}$ of the IRAC position(s).

MCPS data, which we obtained via VizieR, and OGLE-III, which we obtained from their website.

We first searched for a match to each MSX source with the Spitzer data. The program Surveying the Agents of Galactic Evolution (SAGE; Meixner et al. 2006) mapped the LMC and was expanded with the SAGE-SMC program to the SMC (Gordon et al. 2011). We updated the coordinates for each source if it was detected by the Infrared Array Camera (IRAC; Fazio et al. 2004), and failing that, detected at $24 \mu \mathrm{m}$ with the Multiband Imaging Photometer for Spitzer (MIPS; Rieke et al.
2004). We then searched the 2MASS catalog with the new coordinates and updated with them when we found a match.

In four cases (040, 083, 094, and 220), the larger MSX beam included two IRAC sources with a magnitude difference of one or less at $8 \mu \mathrm{m}$. We split these four pairs into separate sources labeled "A" and "B," giving us 247 sources in all. Only seven of the MSX sources have no viable 2MASS counterpart. Three have IRAC positions, three have MIPS positions, and one, MSX SMC 032, has no point source counterpart at all. For this one source, we retained its MSX position. 
Table 6

MSX SMC Source Photometry: Mid-IR

\begin{tabular}{|c|c|c|c|c|c|c|c|}
\hline $\begin{array}{l}\text { MSX } \\
\text { SMC }\end{array}$ & $\begin{array}{l}{[3.6]^{\mathrm{a}}} \\
(\mathrm{mag})\end{array}$ & $\begin{array}{l}{[4.5]^{\mathrm{a}}} \\
(\mathrm{mag})\end{array}$ & $\begin{array}{l}{[5.8]} \\
(\mathrm{mag})\end{array}$ & $\begin{array}{l}{[8.0]} \\
\text { (mag) }\end{array}$ & $\begin{array}{c}{[12]} \\
(\mathrm{mag})\end{array}$ & $\begin{array}{c}{[22]} \\
(\mathrm{mag})\end{array}$ & $\begin{array}{c}{[24]} \\
(\mathrm{mag})\end{array}$ \\
\hline 100 & $12.469 \pm 0.046$ & $11.922 \pm 0.168$ & $10.033 \pm 0.025$ & $8.188 \pm 0.039$ & $6.419 \pm 0.033$ & $2.527 \pm 0.026$ & $2.353 \pm 0.021$ \\
\hline 101 & $8.402 \pm 0.105$ & $8.402 \pm 0.064$ & $8.190 \pm 0.092$ & $7.914 \pm 0.089$ & $7.435 \pm 0.024$ & $5.936 \pm 0.111$ & $5.942 \pm 0.060$ \\
\hline 103 & $5.908 \pm 0.251$ & $6.076 \pm 0.143$ & $6.076 \pm 0.027$ & $6.037 \pm 0.007$ & $6.138 \pm 0.110$ & $6.010 \pm 0.097$ & $5.983 \pm 0.011$ \\
\hline 104 & $11.195 \pm 0.409$ & $9.149 \pm 0.075$ & $7.867 \pm 0.041$ & $6.820 \pm 0.042$ & $5.642 \pm 0.020$ & $2.481 \pm 0.021$ & $2.290 \pm 0.118$ \\
\hline 105 & $9.244 \pm 0.227$ & $8.483 \pm 0.224$ & $8.007 \pm 0.079$ & $7.370 \pm 0.078$ & $6.787 \pm 0.019$ & $6.012 \pm 0.089$ & $5.928 \pm 0.220$ \\
\hline
\end{tabular}

Note. The sources start with MSX SMC 100 for consistency with Table 5.

${ }^{a}$ No color corrections were made when combining the IRAC and WISE data (at 3.4-3.6 $\mu \mathrm{m}$ and $4.5-4.6 \mu \mathrm{m}$ ).

(This table is available in its entirety in machine-readable form.)

Table 7

MSX SMC Source Photometry: Optical and Near-IR

\begin{tabular}{lcccrrrr}
\hline \hline $\begin{array}{l}\text { MSX } \\
\text { SMC }\end{array}$ & $\begin{array}{c}U^{\mathrm{a}} \\
(\mathrm{mag})\end{array}$ & $\begin{array}{c}B \\
(\mathrm{mag})\end{array}$ & $\begin{array}{c}V \\
(\mathrm{mag})\end{array}$ & \multicolumn{1}{c}{$\begin{array}{c}I \\
(\mathrm{mag})\end{array}$} & \multicolumn{1}{c}{$\begin{array}{c}J \\
(\mathrm{mag})\end{array}$} & \multicolumn{1}{c}{$\begin{array}{c}K_{s} \\
(\mathrm{mag})\end{array}$} \\
\hline 100 & $\ldots$ & $\ldots$ & $\ldots$ & $16.443 \pm 0.090$ & $15.398 \pm 0.195$ & $15.101 \pm 0.243$ & $14.350 \pm 0.226$ \\
101 & $\ldots$ & $\ldots$ & $\ldots$ & $10.878 \pm 0.030$ & $9.642 \pm 0.052$ & $8.897 \pm 0.097$ & $8.579 \pm 0.028$ \\
102 & $\ldots$ & $\ldots$ & $\ldots$ & $20.239 \pm 0.021$ & $16.569 \pm 0.657$ & $14.059 \pm 0.664$ & $11.741 \pm 0.499$ \\
103 & $\ldots$ & $\ldots$ & $\ldots$ & $8.939 \pm 0.020$ & $7.071 \pm 0.180$ & $6.342 \pm 0.008$ & $5.794 \pm 0.495$ \\
104 & $18.601 \pm 0.074$ & $18.959 \pm 0.048$ & $18.330 \pm 0.111$ & $17.549 \pm 0.132$ & $16.616 \pm 0.283$ & $15.736 \pm 0.193$ & $14.155 \pm 0.156$ \\
105 & $\cdots$ & $\ldots$ & $21.099 \pm 9.999$ & $18.370 \pm 0.167$ & $15.151 \pm 0.446$ & $13.082 \pm 0.382$ & $11.245 \pm 0.290$ \\
\hline
\end{tabular}

Note. The sources start with MSX SMC 100 for consistency with Table 5.

${ }^{a}$ An uncertainty of 9.999 indicates that only a single measurement was available for a given star in that band.

(This table is available in its entirety in machine-readable form.)

The left panel of Figure 12 compares the positions in the MSX catalog to the IRAC positions from the SAGE-SMC survey, and the right panel compares the IRAC positions to 2MASS. The median offset between the original MSX positions and the corresponding IRAC source is 2 !" 1 , with $90 \%$ of the sources within 5 !" 0 . The median offset compares favorably to the quoted semimajor axis of the typical positional error ellipse, $\sim 2$ ". 3 (Egan et al. 2003). The IRAC and 2MASS positions are significantly closer, with a median separation of 0 . $12,90 \%$ of the sources within 0. " 30 , and all of the matching sources within 1 ". 25 . These values are a testament to the astrometric precision of both the 2MASS and SAGE-SMC surveys.

Table 5 gives the positions and a reference for all 247 sources. It also provides a grade assessing the complexity of the field around each source, based on a comparison of nearby sources in other catalogs and an examination of the images at $8 \mu \mathrm{m}$ (IRAC), $12 \mu \mathrm{m}$ (WISE), and $24 \mu \mathrm{m}$ (MIPS). Of the 247 sources considered, 197 have a grade of "A," which means that they are isolated point sources with little or no extended emission around them. The 36 sources with a "B" grade have an IRAC point source within $20^{\prime \prime}$ or extended emission which contaminates the MSX beam. Grades " $C$ " to "F" describe increasingly complex or crowded fields, with eight grade " $\mathrm{C}$ " sources and two with grade "D." Three sources are grade "E": MSX SMC 023, 068, and 176. MSX SMC 032, with no pointlike object in its vicinity at any wavelength, is the only " $F$ " source. Both MSX SMC 023 and 032 are part of the NGC 248 LHA 115-N 13 star-forming complex.
Figure 13 provides thumbnail sketches from MSX, IRAC, MIPS, and WISE to illustrate some of the complex fields in the SMC. In the top row, the two "worst" MSX sources (MSX SMC 023 on the right and 032 on the left) resolve into bright parts of NGC 248 LHA 115-N 13. The other rows show grade B sources. For MSX SMC 039, in the second row, an extended source is lurking to the northeast, which contributes $\sim 25 \%$ of the flux at $8 \mu \mathrm{m}$. MSX SMC 094 has been resolved by IRAC (and 2MASS) into two nearly equal sources. MSX SMC 125 is a carbon star, but a nearby object $\left(\sim 10^{\prime \prime}\right)$, [M2002] SMC 21202 , contributes about $25 \%$ of the flux at $8 \mu \mathrm{m}$ and dominates the area at $24 \mu \mathrm{m}$. The IRS peaked up on this object rather than the intended target. Fortuitously, both objects were in the SL slit, so those data for MSX SMC 125 could be included in the carbon star overview study of Sloan et al. (2016).

For the sources with IRS spectra, Table 5 also includes the bolometric magnitude calculated by combining the spectral data with the photometry from the next section. The type of object based on the IRS spectrum is given, along with a reference for the classification and additional analysis. The last column indicates the objects that are likely foreground stars (FG), based on having a non-zero proper motion or parallax in Simbad, or are members of a cluster (CL), also from Simbad.

\section{A.2. Mean Photometry}

The mid-IR surveys provide excellent multiple-epoch coverage. The SAGE-SMC survey provides two epochs in all filters in 2007-2008, and it incorporates the Spitzer Survey of the 
SMC ( $\mathrm{S}^{3} \mathrm{MC}$ or S3MC; Bolatto et al. 2007), which provided an earlier epoch for the core of the SMC in 2004. The SAGEVAR program followed up with four more epochs in the core of the SMC from 2010 to 2011 ("VAR" refers to the focus of this program on variable stars; Riebel et al. 2015). The WISE data include the reactivated mission to study near-Earth objects (NEOWISE-R; Mainzer et al. 2014). The all-sky scanning strategy for WISE covers the SMC every six months, giving two epochs in 2010 and four in the period 2014-2015. Thus we have up to 13 mid-IR epochs in the core of the SMC and up to 8 in the outskirts.

Table 6 presents the mean magnitudes in the mid-IR for each MSX source. We combined IRAC data at $3.6 \mu \mathrm{m}$ with the WISE data at $3.4 \mu \mathrm{m}$ (W1) without making color corrections. We combined IRAC $4.5 \mu \mathrm{m}$ data with WISE $4.6 \mu \mathrm{m}$ data (W2) similarly. Sloan et al. (2016) calibrated color corrections between these filter pairs for carbon stars, but the present MSX sample contains many other source types. The greater overlap between $\mathrm{W} 2$ and the $4.5 \mu \mathrm{m}$ IRAC filter leads to smaller corrections compared to $\mathrm{W} 1$ and $3.6 \mu \mathrm{m}$.

Several epochs are also available in the near-IR. The 2MASS maps at $J, H$, and $K_{s}$ were obtained in 1998 August and followed up with a deeper survey, known as 2MASS 6X, in 2000 December. DENIS adds epochs at $I, J$, and $K_{s}$ from 1996 to 1999 , and the IRSF adds more at $J, H$, and $K_{s}$ from 2002 to 2006. Table 7 presents the mean magnitudes for each filter in the near-IR. We have not attempted to account for subtle differences between the filter sets from the different telescopes. The MCPS provides mean magnitudes at $U, B, V$, and $I$. When mean magnitudes at $V$ and $I$ are available from the LPV catalogs from OGLE-III, we substituted these for the MCPS values. These mean optical and near-IR magnitudes are given in Table 7.

\section{REFERENCES}

Beichman, C. A., Rieke, M., Eisenstein, D., et al. 2012, Proc. SPIE, 8442, $84422 \mathrm{~N}$

Beichman, C. A., Neugebauer, G., Habing, H. J. et al. (ed.) 1988, Infrared Astronomical Satellite (IRAS) Catalogs and Atlases, Vol. 1: Explanatory Supplement (Washington, DC: NASA)

Bernard-Salas, J., Peeters, E., Sloan, G. C., et al. 2006, ApJL, 652, L29

Bernard-Salas, J., Peeters, E., Sloan, G. C., et al. 2009, ApJ, 699, 1541

Bernard-Salas, J., Pottasch, S. R., Gutenkunst, S., Morris, P. W., \& Houck, J. R. 2008, ApJ, 672, 274

Blanco, V. M., McCarthy, M. F., \& Blanco, B. M. 1980, ApJ, 242, 938

Bloecker, T., \& Schoenberner, D. 1991, A\&A, 244, L43

Bolatto, A. D., Simon, J. D., Stanimirović, S., et al. 2007, ApJ, 655, 212

Bouchet, P., García-Marín, M., Lagage, P.-O., et al. 2015, PASP, 127, 612

Boyer, M. L., Srinivasan, S., van Loon, J. T., et al. 2011, AJ, 142, 103

Castilho, B. V., Gregorio-Hetem, J., Spite, F., Spite, M., \& Barbuy, B. 1998, A\&AS, 127, 139

Cioni, M.-R., Loup, C., Habing, H. J., et al. 2000, A\&AS, 144, 235

Cutri, R. M., Skrutskie, M. F., van Dyk, S., et al. 2003, 2MASS All Sky Catalog of Point Sources (Washington, DC: NASA)

D'Antona, F., \& Mazzitelli, I. 1996, ApJ, 470, 1093

Egan, M. P., Price, S. D., Kraemer, K. E., et al. 2003, The Midcourse Space Experiment Point Source Catalog, Version 2.3, Tech. Rep. AFRL-VS-TR2003-1589 (Fort Belvoir, VA: DTIC) http://www.dtic.mil/docs/citations/ ADA418993

Egan, M. P., \& Sloan, G. C. 2001, ApJ, 558, 165

Egan, M. P., Van Dyk, S. D., \& Price, S. D. 2001, AJ, 122, 1844

Elias, J. H., Frogel, J. A., \& Humphreys, R. M. 1980, ApJL, 242, L13

Elias, J. H., Frogel, J. A., \& Humphreys, R. M. 1985, ApJS, 57, 91

Engelke, C. W. 1992, AJ, 104, 1248

Fazio, G. G., Hora, J. L., Allen, L. E., et al. 2004, ApJS, 154, 10

Gielen, C., Bouwman, J., van Winckel, H., et al. 2011, A\&A, 533, A99
Gielen, C., van Winckel, H., Min, M., Waters, L. B. F. M., \& Lloyd Evans, T. 2008, A\&A, 490, 725

González-Fernández, C., Dorda, R., Negueruela, I., \& Marco, A. 2015, A\&A, 578, A3

Gordon, K. D., Meixner, M., Meade, M. R., et al. 2011, AJ, 142, 102

Groenewegen, M. A. T. 2004, A\&A, 425, 595

Groenewegen, M. A. T., \& Blommaert, J. A. D. L. 1998, A\&A, 332, 25

Groenewegen, M. A. T., Sloan, G. C., Soszyński, I., \& Petersen, E. A. 2009, A\&A, 506, 1277

Gruendl, R. A., Chu, Y.-H., Seale, J. P., et al. 2008, ApJL, 688, L9 Guha Niyogi, S., Speck, A. K., \& Onaka, T. 2011, ApJ, 733, 93

Houck, J. R., Roellig, T. L., van Cleve, J., et al. 2004, ApJS, 154, 18 Jones, O. C., Kemper, F., Sargent, B. A., et al. 2012, MNRAS, 427, 3209

Jones, O. C., Kemper, F., Srinivasan, S., et al. 2014, MNRAS, 440, 631

Jones, O. C., Meixner, M., Justtanont, K., \& Glasse, A. 2016, ApJ, submitted Jones, O. C., Meixner, M., Sargent, B. A., et al. 2015, ApJ, 811, 145

Kato, D., Nagashima, C., Nagayama, T., et al. 2007, PASJ, 59, 615

Keller, S. C., \& Wood, P. R. 2006, ApJ, 642, 834

Kraemer, K. E., Price, S. D., Mizuno, D. R., \& Carey, S. J. 2002a, AJ, 124, 2990

Kraemer, K. E., Shipman, R. F., Price, S. D., et al. 2003, AJ, 126, 1423

Kraemer, K. E., Sloan, G. C., Bernard-Salas, J., et al. 2006, ApJL, 652, L25

Kraemer, K. E., Sloan, G. C., Price, S. D., \& Walker, H. J. 2002b, ApJS, 140, 389

Kraemer, K. E., Sloan, G. C., Wood, P. R., Price, S. D., \& Egan, M. P. 2005, ApJL, 631, L147

Kwon, Y.-J., \& Suh, K.-W. 2014, JKAS, 47, 123

Lagadec, E., Zijlstra, A. A., Sloan, G. C., et al. 2007, MNRAS, 376, 1270

Lebouteiller, V., Bernard-Salas, J., Sloan, G. C., \& Barry, D. J. 2010, PASP, 122,231

Levesque, E. M., Massey, P., Olsen, K. A. G., et al. 2006, ApJ, 645, 1102

Li, A., \& Draine, B. T. 2002, ApJ, 572, 232

Lloyd Evans, T. 1990, MNRAS, 243, 336

Lloyd Evans, T. 1995, Ap\&SS, 230, 169

Lloyd Evans, T. 2010, JApA, 31, 177

Mainzer, A., Bauer, J., Cutri, R. M., et al. 2014, ApJ, 792, 30

Marigo, P. 1998, A\&A, 340, 463

Massey, P., \& Olsen, K. A. G. 2003, AJ, 126, 2867

McDonald, I., Sloan, G. C., Zijlstra, A. A., et al. 2010, ApJL, 717, L92

Meixner, M., Gordon, K. D., Indebetouw, R., et al. 2006, AJ, 132, 2268

Molster, F. J., Yamamura, I., Waters, L. B. F. M., et al. 1999, Natur, 401, 563

Neugebauer, G., Habing, H. J., van Duinen, R., et al. 1984, ApJL, 278, L1

Oliveira, J. M., van Loon, J. T., Sloan, G. C., et al. 2013, MNRAS, 428, 3001

Paczyński, B. 1971, AcA, 21, 417

Pojmański, G. 1997, AcA, 47, 467

Pojmański, G. 2014, CoSka, 43, 523

Polsdofer, E., Seale, J., Sewiło, M., et al. 2015, AJ, 149, 78

Prevot, L., Martin, N., Rebeirot, E., Maurice, E., \& Rousseau, J. 1983, A\&AS, 53, 255

Price, S. D., Egan, M. P., Carey, S. J., Mizuno, D. R., \& Kuchar, T. A. 2001, AJ, 121, 2819

Raimondo, G., Cioni, M.-R. L., Rejkuba, M., \& Silva, D. R. 2005, A\&A, 438,521

Rebeirot, E., Azzopardi, M., \& Westerlund, B. E. 1993, A\&AS, 97, 603

Riebel, D., Boyer, M. L., Srinivasan, S., et al. 2015, ApJ, 807, 1

Rieke, G. H., Wright, G. S., Böker, T., et al. 2015, PASP, 127, 584

Rieke, G. H., Young, E. T., Engelbracht, C. W., et al. 2004, ApJS, 154, 25

Rubele, S., Girardi, L., Kerber, L., et al. 2015, MNRAS, 449, 639

Ruffle, P. M. E., Kemper, F., Jones, O. C., et al. 2015, MNRAS, 451, 3504

Samus, N. N., Durlevich, O. V., et al. 2009, yCat, 1, 2025

Sanduleak, N. 1989, AJ, 98, 825

Sargent, B. A., Srinivasan, S., \& Meixner, M. 2011, ApJ, 728, 93

Skrutskie, M. F., Cutri, R. M., Stiening, R., et al. 2006, AJ, 131, 1163

Sloan, G. C., Devost, D., Bernard-Salas, J., Wood, P. R., \& Houck, J. R. 2006a, ApJ, 638, 472

Sloan, G. C., Herter, T. L., Charmandaris, V., et al. 2015, AJ, 149, 11

Sloan, G. C., Kraemer, K. E., Matsuura, M., et al. 2006b, ApJ, 645, 1118

Sloan, G. C., Kraemer, K. E., McDonald, I., et al. 2016, ApJ, 826, 44

Sloan, G. C., Kraemer, K. E., Wood, P. R., et al. 2008, ApJ, 686, 1056

Sloan, G. C., Lagadec, E., Zijlstra, A. A., et al. 2014, ApJ, 791, 28

Sloan, G. C., \& Price, S. D. 1995, ApJ, 451, 758

Sloan, G. C., \& Price, S. D. 1998, ApJS, 119, 141

Smith, V. V., Plez, B., Lambert, D. L., \& Lubowich, D. A. 1995, ApJ, 441, 735

Soszyński, I., Udalski, A., Szymański, M. K., et al. 2011, AcA, 61, 217

Speck, A. K., Cami, J., Markwick-Kemper, C., et al. 2006, ApJ, 650, 892

Sylvester, R. J., Barlow, M. J., \& Skinner, C. J. 1994, MNRAS, 266, 640 
Sylvester, R. J., Skinner, C. J., \& Barlow, M. J. 1998, MNRAS, 301, 1083 Szczerba, R., Siódmiak, N., Stasińska, G., \& Borkowski, J. 2007, A\&A, 469, 799

Trams, N. R., van Loon, J. T., Zijlstra, A. A., et al. 1999, A\&A, 344, L17 van Loon, J. T. 2012, arXiv: 1210.0983

van Loon, J. T., Cohen, M., Oliveira, J. M., et al. 2008, A\&A, 487, 1055

Verhoelst, T., van der Zypen, N., Hony, S., et al. 2009, A\&A, 498, 127

Wainscoat, R. J., Cohen, M., Volk, K., Walker, H. J., \& Schwartz, D. E. 1992, ApJS, 83, 111

Werner, M. W., Roellig, T. L., Low, F. J., et al. 2004, ApJS, 154, 1

Whitelock, P. A., Feast, M. W., Menzies, J. W., \& Catchpole, R. M. 1989, MNRAS, 238, 769
Whitney, B. A., Wood, K., Bjorkman, J. E., \& Cohen, M. 2003, ApJ, 598, 1079

Wong, G. F., Crawford, E. J., Filipovic, M. D., et al. 2012, SerAJ, 184, 93

Wood, P. R., Bessell, M. S., \& Fox, M. W. 1983, ApJ, 272, 99

Woods, P. M., Oliveira, J. M., Kemper, F., et al. 2011, MNRAS, 411, 1597

Wright, E. L., Eisenhardt, P. R. M., Mainzer, A. K., et al. 2010, AJ, 140, 1868

Yamamura, I., Dominik, C., de Jong, T., Waters, L. B. F. M., \& Molster, F. J. 2000, A\&A, 363, 629

Zaritsky, D., Harris, J., Thompson, I. B., Grebel, E. K., \& Massey, P. 2002, AJ, 123,855

Zijlstra, A. A., Matsuura, M., Wood, P. R., et al. 2006, MNRAS, 370, 1961 